\title{
La gestion des partenaires d'échange et des objets occidentaux par les chefs fidjiens (1800-1855)
}

Fijian Chiefs' Management of Exchange Partners and Western Objects (18001855)

\section{Stéphanie Leclerc-Caffarel}

\section{(2) OpenEdition}

\section{Journals}

Édition électronique

URL : http://journals.openedition.org/jso/7338

DOI : $10.4000 /$ jso.7338

ISSN : $1760-7256$

Éditeur

Société des océanistes

Édition imprimée

Date de publication : 15 décembre 2015

Pagination : 199-222

ISBN : 978-2-85430-126-7

ISSN : 0300-953x

\section{Référence électronique}

Stéphanie Leclerc-Caffarel, « La gestion des partenaires d'échange et des objets occidentaux par les chefs fidjiens (1800-1855) », Journal de la Société des Océanistes [En ligne], 141 | juillet-décembre 2015, mis en ligne le 15 décembre 2018, consulté le 22 avril 2019. URL : http://journals.openedition.org/ jso/7338; DOl : 10.4000/jso.7338 


\section{La gestion des partenaires d'échange et des objets occidentaux par les chefs fidjiens (1800-1855)}

par

Stéphanie LECLERC-CAFFAREL*

\section{RÉSUMÉ}

Dès 1800, un grand nombre de navires occidentaux visitent les îles Fidji pour des raisons commerciales, scientifiques et religieuses. Avant 1855, ces visiteurs auraient eu peu d'impact direct sur l'équilibre politique de l'archipel, asservi à la diplomatie de quelques grandes chefferies. Dans les conflits locaux néanmoins, des changements sopèrent. La place des armes à feu et de ceux qui savent les manier devient cruciale, alors même que d'autres objets d'importation comme les dents de cachalot prennent de l'importance sur les plans politique et symbolique. À ceci s'ajoute l'installation des premiers missionnaires chrétiens dans les îles. Sintéressant à la gestion de ces intrusions étrangères par les chefs fidjiens, avec une attention particulière aux chefferies de Bua, Bau, Lau et Somosomo, cet article illustre en quoi l'intégration des biens et des hommes étrangers à la communauté relève à la fois de dynamiques déjà à l'ouvre, de catégories fidjiennes préexistantes et d'influences extérieures. Surtout, il souligne l'importance des échanges dans ces relations.

Mots-CLÉs : Fidji, XIX siècle, voyageurs, biens d'importation, échanges, systèmes de valeur

Routledge (1985 : 5-6) explique que la cession de la souveraineté fidjienne à la couronne d'Angleterre (1874) résulte au moins en partie d'une interférence des intérêts étrangers - occidentaux

\begin{abstract}
From 1800 on, a number of Western vessels visited the Fiji islands for commercial, scientific or religious reasons. Before 1855, such visitors seem to have had little impact on the political balance of the archipelago, which depended on a few powerful chiefdoms. In local conflicts, however, things were changing. The role of firearms and of those who knew how to handle them became crucial, while other imported goods such as whale's teeth were growing a political and symbolic significance. To this, one must add the settlement of the first Christian missionaries in the islands. This article is concerned with the chiefly management of these foreign intrusions. With specific attention to the chiefdoms of Bua, Bau, Lau and Somosomo, it shows that the absorption of outsider men and goods into the Fijian community fell under local dynamics, pre-existing Fijian categories as well as foreign influences. Above all, it highlights the importance of exchanges within these relations.
\end{abstract}

KeYwords: Fiji, $19^{\text {th }}$ century, voyagers, imported goods, exchanges, value systems

notamment - dans la politique de la région ${ }^{1}$. Pour la période antérieure à 1855 , il insiste davantage sur les luttes de pouvoir entre les grandes chefferies de l'archipel, et sur leur rôle dans la construc-

1. Le terme « Fidji » et ses dérivés, en ce qu'ils renvoient à une entité cohérente et à des frontières stables, sont anachroniques ici (Leclerc-Caffarel, 2013 : 2). Ils désignent dans cet article l'aire géographique correspondant à l'État moderne de la république de Fidji, à l'exception de Rotuma dont l'ajout historique au groupe en 1879 n'est pas pertinent pour mon propos (voir aussi Leclerc-Caffarel, $2013:$ 41-42). Il convient toutefois de replacer Fidji à la frontière de la Polynésie occidentale et dans le contexte géopolitique du XIx e siècle, dans l'aire d'influence de Tonga et de la colonie britannique de Port Jackson (Australie). Le mot "Occident " et ses dérivés désignent l'Europe et les États-Unis d'Amérique, d'un point de vue géographique et politique, ainsi que les colons d'origine européenne présents en Australie et en Nouvelle-Zélande au XIX ${ }^{e}$ siècle.

* Post-doctorante au musée du quai Branly et associée au Fiji Museum, stephanie.caffarel@gmail.com 


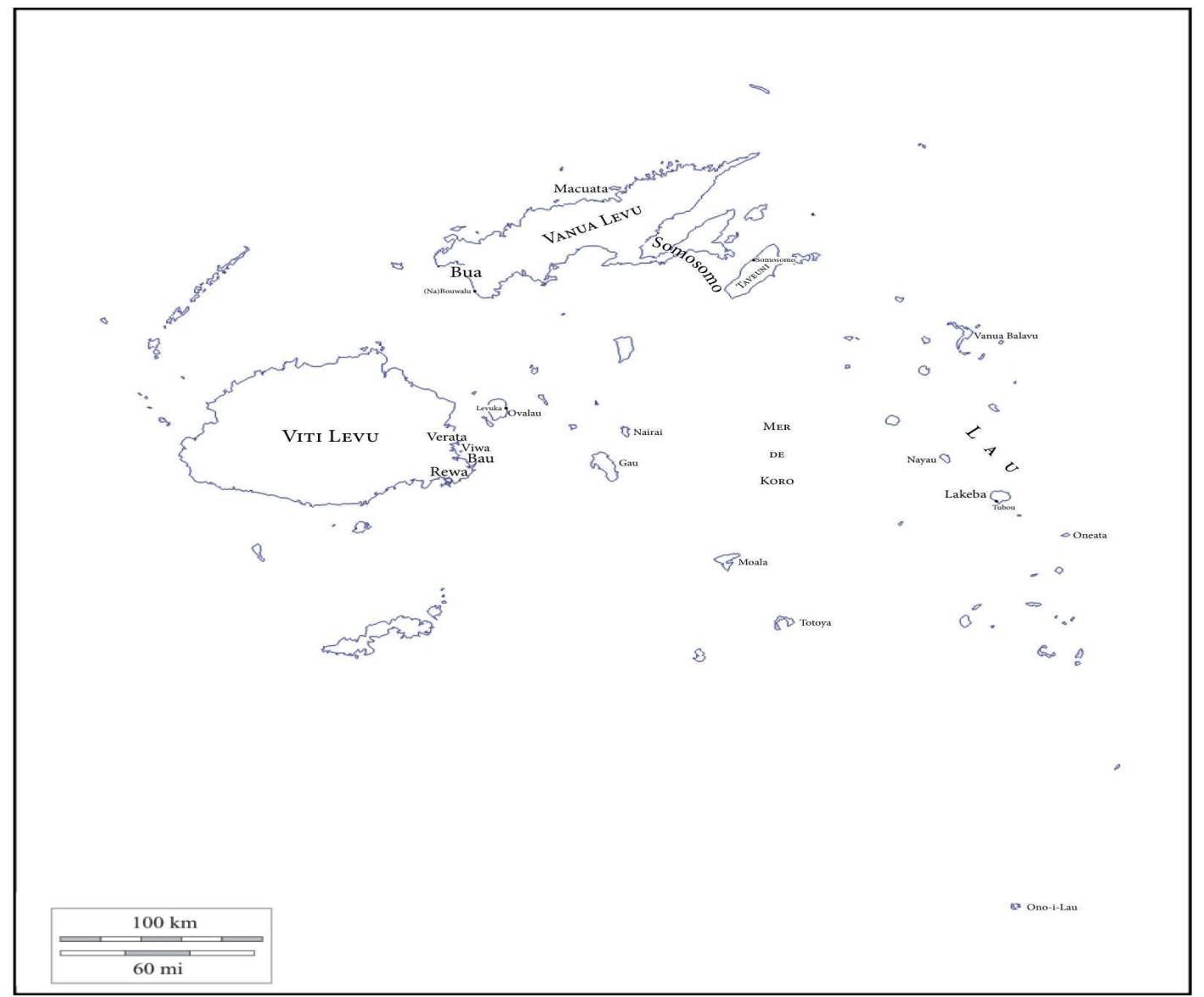

CARTe 1. - Les îles Fidji au XIX $x^{e}$ siècle, mentionnant les principales chefferies citées dans le texte (carte de l'auteur)

tion des Fidji modernes. Les Euro-Américains ne peuvent pas encore être considérés comme des acteurs indépendants de la politique fidjienne (idem: 36-38). Néanmoins, au début du $\mathrm{XIX}^{\mathrm{e}}$ siècle, la place des armes à feu et de ceux qui savent les manier devient cruciale (ibid. : 43-48). D'autres objets d'importation comme les dents de cachalot prennent aussi de l'importance sur les plans politique et symbolique. Et dès les années 1830, les missionnaires chrétiens viennent ajouter le lotu (christianisme) ${ }^{2}$ aux enjeux diplomatiques préexistants. La question du rôle des chefs dans la gestion de ces intrusions étrangères est centrale. Comment, et selon quelles modalités, des objets et des hommes tels que les armes à feu et les beachcombers (écumeurs de grève) entrentils dans le quotidien des Fidjiens au XIX ${ }^{e}$ siècle? Dans quelle mesure les chefs en font-ils des instruments de leur propre ascension, plutôt qu'ils n'en sont les victimes ? Peut-on généraliser à tout l'archipel des motifs d'intégration qui pourraient n'être le fait que d'une chefferie, voire d'un chef ? Parce qu'ils sont les interlocuteurs principaux des voyageurs occidentaux avant 1855 , cet article se place du côté des chefs (turaga), c'est-à-dire des figures d'autorité politique et religieuse ${ }^{3}$ telles que les définissent notamment Hooper (1996) dans les Lau et Sahlins (1985) historiquement. En particulier, il s'intéresse aux chefs des rangs les plus élevés que je distingue ici des chefs dont l'autorité est plus localisée en les appelant Turaga, avec une majuscule. Par contre, cet article fait une relative abstraction du "peuple». Il faut pourtant garder à l'esprit que les rôles et les statuts des Turaga se définissent par rapport à ceux du Vanua - le territoire et sa population - qui est par essence l'entité complémentaire et partenaire des Turaga $a^{4}$. Dans les régions de l'est et du nord de Fidji que

2. Le mot lotu, d'origine tongienne, désigne la religion chrétienne et ses différentes branches.

3. En matière de religion aussi, il convient de noter le rôle des chefs. Comme le formule Hocart : « [...] there is no religion in Fiji, only a system which in Europe has split into religion and business" (1970 : 256). Par essence, la personne du Turaga est sacrée $(t a b u)$ et l'ordonnancement du monde autour d'elle duel, quoiqu'il y ait des subtilités dans la répartition des rôles spirituels et temporels dans certaines chefferies comme celle de Bau (Hocart, 1970 : 163-164).

4. Il est très difficile de généraliser à Fidji, en particulier au début du XIX ${ }^{\mathrm{e}}$ siècle. Larchipel est alors extrêmement morcelé sur le plan politique. Néanmoins, les cas d'étude choisis ici sont relativement proches du point de vue de leur fonctionnement social et politique, pour autant qu'on puisse en juger par les sources anciennes à notre disposition. Ils sont aussi apparentés en termes linguistiques (Geraghty, 1983). Le lecteur est cependant prié de garder à l'esprit l'absence d'unité 
cet article considère, cette relation réciproque (veiqaravi) vis-à-vis du Vanua repose notamment sur des échanges - de femmes, d'objets de valeur (iyau) et de nourriture - attestés dans l'ethnographie contemporaine comme dans les mythes et les récits historiques (par exemple, Hooper, 1982, 1996 ; Sahlins, 1983, 1985, 2004) . En fait, une telle dualité peut être formulée pour tout binôme itaukei (autochtones) vulagi (étrangers, invités); la relation Turaga-Vanua est un cas particulier (Hooper, 1996: 247, 264 ; Toren, 1994). Les chefs fidjiens des rangs les plus élevés sont considérés comme étrangers à la terre sur laquelle ils règnent (Sahlins, 1985 : 78). Ils s'allient au peuple autochtone par voie de mariage(s) et d'échanges et sont assimilés à des dieux locaux. Puisque c'est le binôme chefs fidjiens-voyageurs occidentaux qui nous intéresse ici, il convient de considérer à leur tour les Euro-Américains comme des "partenaires d'échange" des Turaga. Dans cette configuration, les premiers sont plus étrangers au Vanua que les seconds. Cet article interroge le rôle et la nature de leurs échanges.

Les termes fidjiens mentionnés plus haut sont polysémiques. En fidjien standard aujourd'hui, "turaga " désigne les individus de sexe masculin ${ }^{6}$ que le rang et/ou la situation distinguent. Selon le contexte, il s'agit d'une formule respectueuse proche de l'anglais gentleman ou d'un indicateur de statut social. Je traduis ici turaga par " chef». Ce terme s'applique à un grand nombre d'individus à Fidji selon les subdivisions considérées, et avec des variations régionales, historiques et circonstancielles importantes (Hooper, 1996). Les chefs principaux de Fidji se distinguent par leur position sociale, leurs prérogatives cérémonielles, la part d'essence divine qu'on leur attribue et leur influence étendue, mais aussi par leur rapport à la mer - à l'extérieur des îles (Hooper, 1996, 2013). Les chefs de rangs inférieurs sont à la tête d'unités sociales plus réduites. Celles-ci incluent des groupes familiaux (tokatoka) et des clans (mataqali), constitutifs du Vanua $^{7}$ ou de groupes satellites qui lui sont associés - clans guerriers par exemple. Plus le rang et les fonctions sont importants, plus le chef qui les incarne assume de niveaux d'autorité. Pour plus de clarté, je m'efforcerai ici d'utiliser les titres fidjiens plutôt que des formules générales. Ainsi, je dirai le Vunivalu de Bau plutôt que le chef de Bau. La notion de chef suprême est fluctuante avant 1855 ; elle est en partie en jeu dans les exemples discutés plus bas ${ }^{8}$.

En résumé, cet article cherche à comprendre comment, avant 1855 , les chefs fidjiens de haut rang (Turaga) intègrent les visiteurs occidentaux ainsi que les biens et les idées qu'ils véhiculent; et comment ceux-ci influencent leur propre représentation du pouvoir. Le développement qui suit consiste en une analyse chronologique d'échanges et de phénomènes d'assimilation dans quatre localités (Bua, Bau, Lau et Somosomo9). Les quatre premières parties mettent chacune l'accent sur une de ces chefferies. La dernière section est consacrée à la conversion au christianisme (lotu) de Cakobau, le Vunivalu de Bau considéré historiquement comme le chef le plus important des Fidji en raison de son rôle dans l'instauration du protectorat britannique en $1874^{10}$.

\section{Bua Bay : le marché du bois de santal}

Après une phase de "découverte " initiée par Tasman (1643) et Cook (1774), les relations entre Fidjiens et Occidentaux prennent un nouvel essor pendant la première partie du $\mathrm{XIX}^{\mathrm{e}}$ siècle. Le bois de santal, les bêches-de-mer ${ }^{11}$ et l'écaille de tortue font l'objet d'un marché lucratif entre le Pacifique, l'Europe, l'Amérique et l'Asie. Fidji se trouve en outre sur la route des baleiniers et des explorateurs cartographes qui parcourent le Pacifique Sud. De

dans ces domaines. Tous les termes fidjiens employés ici sont en fidjien standard, issu du dialecte de Bau - l'une des trois langues officielles à Fidji aujourd'hui avec l'anglais et l'hindi.

5. Concernant le principe de réciprocité, impérieux dans plusieurs domaines de la vie fidjienne contemporaine, voir aussi Ravuvu (1983: 11).

6. Marama est l'équivalent féminin.

7. Comme turaga, vanua est un terme polysémique, avec plusieurs niveaux de signification selon le contexte. Ceux-ci sont bien explicités par Hooper pour les Lau (1996 : 247). Vanua avec une majuscule désigne ici le territoire principal sur lequel règne un Turaga, ainsi que ses habitants. Il s'agit d'une entité géopolitique, par opposition au vanua (lieu) qui renvoie à une localisation géographique. Il s’oppose aussi aux territoires assujettis ou tributaires (qali).

8. Les personnages fidjiens mentionnés dans le texte, ainsi que les titres principaux pour chaque chefferie, sont repris dans un tableau récapitulatif placé en annexe.

9. La ville de Somosomo (Taveuni) est le siège de la chefferie du Nord, aux mains du clan (mataqali) iSokula depuis le début du XIX ${ }^{e}$ siècle au moins puisque le quatrième Tui Cakau, Yavala, règne de 1829 à 1845. L'apparition du titre Tui Cakau a lui-même son origine dans l'accession au pouvoir des iSokula (Routledge, 1985 : 38). Dans la littérature ancienne, le nom éponyme de Somosomo désigne la chefferie dont le leader porte le titre Tui Cakau. C’est en référence à cette littérature et à la situation géopolitique de la première moitié du XIx ${ }^{e}$ siècle que "Somosomo " est utilisé dans cet article. Cakaudrove est une appellation alternative qui désigne aujourd'hui le tiers sud-est de Vanua Levu, ainsi que les îles de Taveuni, Rabi et Kioa.

10. Toutes les sources écrites utilisées ici sont d'origine occidentale, y compris les citations attribuées à des chefs fidjiens.

11. Comme le bois de santal utilisé dans la fabrication de l'encens, ces mollusques aussi appelés holothuries ou trépangs sont prisés sur les marchés chinois, en raison des vertus médicinales et aphrodisiaques qu’on leur prête. Plusieurs variétés proliferrent dans les eaux fidjiennes au début du XIX ${ }^{e}$ siècle. Pêchées puis séchées à Fidji, certaines sont ensuite acheminées vers la Chine via les Philippines, notamment par des armateurs américains. Ce commerce atteint son apogée à Fidji dans les années 1830. 
nombreux navires européens et américains sillonnent ces eaux, apportant avec eux un grand nombre d'interlocuteurs pour les Turaga ${ }^{12}$.

Les habitants de Bua, au sud-est de Vanua Levu, sont les premiers à entrer en contact prolongé avec les Euro-Américains, en raison de la présence de bois de santal dans cette partie de l'île (Derrick, 1957 : 39-42). C'est le naufrage du schooner Argo, en $1800^{13}$ dans les Lau (Bukatatanoa ou Argo Reef), qui initie les transactions décrites ci-après. La plupart des naufragés gagnent Tonga, y compris peut-être Charles Savage dont nous allons reparler. Exception notable, Oliver Slater reste près de deux ans dans l'archipel. Il y repère de grandes quantités de bois de santal. En 1804, il revient l'exploiter (Im Thurn et Wharton, 1925 : XXXII-XXXIX, XLII).

L'exploitation du bois de santal, puis la pêche et le conditionnement des holothuries sont des activités demandeuses de main d'œuvre. Au début du XIX ${ }^{e}$ siècle, les compétences et le nombre d'hommes requis obligent les marins occidentaux à employer des travailleurs autochtones pour réunir des cargaisons conséquentes de bois de santal puis de bêchesde-mer séchées. Et ceci ne peut se faire que par l'entremise des chefs, car leur statut leur permet de requérir ou de réquisitionner un certain nombre de biens et de services auprès de leurs sujets, de leurs parents et de leurs alliés. Les deux formes principales de ces requêtes sont dites kerekere et lala. Elles sont associées au concept veiqali qui désigne le lien entre les sujets et leur chef (Capell, 1941 : 189; Gatty, 2009: 187), matérialisé par un ensemble de prestations. Il exclut par définition les visiteurs euro-américains, étrangers à ce système.

Quoique souvent traduit par " mendier " (Sahlins, $1962: 203 ; 1993: 855-859)$, kerekere désigne en fait une requête justifiée et mutuellement consentie (Sahlins, 1962: 203-214; 1993: 854-855). Cette requête, souvent tacite, permet notamment au vasu (enfant de sœur) de solliciter l'aide ou la propriété de ses parents maternels (Pauwels, 2015 : 144). Quoiqu'elle ne soit pas l'apanage des chefs, cette prérogative prend toute son ampleur dans les hautes sphères de la société fidjienne, puisque l'aide ou les biens en question y sont plus importants.

Le terme lala désigne le travail ou les biens que les gens du commun doivent fournir à leur Turaga, ou la requête de ce dernier pour les obtenir (Capell, 1941 : 130 ; Gatty, 2009 : 130). Pendant la première moitié du XIx ${ }^{e}$ siècle, le lala pouvait prendre plusieurs formes depuis la culture de la terre jusqu'à la construction des maisons, en passant par la pêche - y compris d'holothuries - et l'abattage du bois de santal.

De plus, les Turaga perçoivent des contributions de chefferies tributaires (qali), sous la forme d'objets de valeur et d'échange (iyau $\left.{ }^{14}\right)$, de denrées et de services ${ }^{15}$. Ils sont aussi en droit d'exiger une réparation rituelle (soro) lorsqu'ils ont été offensés $^{16}$. En général, les événements liés au Turaga (mariage, funérailles, installation) brassent un grand nombre de richesses. Par les chefs transitent une grande quantité de biens et de services, selon un système de centralisation (pooling) qui implique une mise en commun des ressources (Sahlins, 1972 : 188-189). Pendant la période qui nous occupe, ce sont les Turaga qui détiennent les clés de l'accès aux richesses fidjiennes, y compris celles que les Occidentaux convoitent et qui permettent d'obtenir des choses importées.

À ces égards, la période de contact entre les marchands de bois de santal et les habitants de Bua ou "Sandalwood" Bay (1800-1815) nous enseigne deux choses essentielles. D'abord, elle montre que les chefs sont les interlocuteurs privilégiés des Euro-Américains - au moins de ceux qui ont la charge des transactions principales et qui les relatent dans leurs carnets de bord. Elle illustre aussi combien les Occidentaux savent tôt s'adapter aux demandes et aux croyances de leurs partenaires autochtones.

\section{Afflux de biens d'importation et rôle des chefs}

À ces deux titres, les écrits de W. Lockerby ${ }^{17}$ sont particulièrement intéressants. En 1808, il relate

12. Des dizaines de capitaines et de logkeepers sont répertoriés par Langdon (1978), pour les bateaux en provenance de la seule côte Est américaine.

13. La date exacte du naufrage fait débat (Derrick, $1957: 39$; Toganivalu, s.d. ; Im Thurn et Warthon, 1925 : xxxIv).

14. La catégorie "iyau » est notamment discutée par Hooper (1982: 50-51) et Thomas (1991: 67-68). Hooper oppose les iyau aux iyaya, choses communes et équipement personnel (gear). Thomas les définit comme des objets précieux voués à être aliénés par l'échange. Aux objets manufacturés listés par Hooper (1982) et Toganivalu (1917), j’ajoute pour ma part un certain nombre d'ornements corporels et d'autres marqueurs de rang (comme les armes), comparables aux " trésors " māori taonga (Nouvelle-Zélande). Quoique ces artefacts ne soient pas toujours destinés à l'échange, ils influencent les transactions en matérialisant les statuts et le mana (efficacité) des protagonistes.

15. Ces taxes (vakacavacava) sont proportionnelles à la puissance de la chefferie, et parfois essentielle à sa subsistance. C'est par exemple le cas de Bau au début du XIx siècle (Gatty, 2009 : 187 ; Sahlins, 2004 : 30-33).

16. Cette pratique existe encore aujourd'hui. Elle prend toute sa mesure lorsqu'elle vise le pardon d'un Turaga puisque les biens impliqués sont plus nombreux et plus prestigieux que ceux nécessaires au règlement d'un différend entre roturiers. Le même terme désignait autrefois la reddition (Gatty, 2009 : 230 ; Capell, 1941 : 237).

17. William Lockerby est l'officier en charge de la cargaison sur le santalier Jenny, stationné à Fidji en mai 1808 (Im Thurn et Wharton, 1925 : LXIV, 11-19). Peu après qu'un premier chargement de santal a été rassemblé, Lockerby est abandonné sur Vanua Levu. Il passe alors plusieurs mois en compagnie des insulaires, sous la protection d'un chef de haut rang de la région de Bua (idem : LXv). De cette cohabitation subsiste un journal publié en 1925 (Im Thurn et Wharton, 1925), et d'autres écrits destinés à ses pairs (Lockerby, 1813 ; Dodge, 1972). 
par exemple comment certaines tentatives pour satisfaire le Tui Bua (voir encadré), basées sur le système de valeur européen, restent vaines :

"The King ${ }^{18}$ [of Bua] had a present made him of a wooden house, by some gentlemen of Botany Bay, Lord, Cable of Co., which he did not like. [...] The King said it would neither keep out the sun, nor the rain. The house was divided into two apartments; one containing the different articles the King had obtained from Europeans, and in the other was deposited the fire-arms that I had with me in the boat, consisting in four muskets, two pairs of pistols, six cutlasses, six boarding pikes, a compass, quadrant, spy-glass, orc. [...]. The King has likewise been presented with a brass laced hat, with a brass crown, but he would much rather have had a whale's tooth, that being the most valuable article among them. They hang them about their necks on great festivals, and give them with their daughters in marriage - as their marriage portion - in short, he who is possessed of a quantity of them, thinks himself extremely rich. " (Im Thurn et Wharton, $1925: 25$ )

Cet extrait montre le manque d'intérêt du Tui Bua pour certains objets de manufacture européenne comme une "couronne en alliage de cuivre »; mais il illustre aussi une attraction pour d'autres biens véhiculés par les Occidentaux, dont les dents de cachalot. Lockerby ne détaille pas la nature des articles " obtenus des Européens " par le chef. D'autres textes permettent néanmoins de penser qu'ils sont similaires à ceux que le subrécargue lui-même entrepose sous la protection du Turaga. Armes à feu et instruments de navigation sont en effet des objets d'échange clés pendant la période qui nous intéresse, tout comme l'ivoire de cachalot décrit ici comme "l'article le plus valorisé parmi eux [natives] $»^{19}$.

Les instructions que Lockerby fournit ensuite à ses pairs (1813) détaillent les moyens à la disposition des marins pour obtenir du bois de santal, par l'intermédiaire des chefs. Le glossaire compilé par le subrécargue fournit la transcription phonétique d'une variété d'objets d'échange, des outils en métal aux miroirs en passant par les perles de verre. Le premier mot de cette liste désigne l'ivoire. Il est traduit par "tamboo ", c'est-à dire probablement tabua - quoique l'homonymie sonore avec le terme tabu (proscrit, sacré) ne puisse être ignorée. Plus loin, Lockerby explique :

" une pièce d'ivoire pesant environ une livre vaut deux tonnes de bois [de santal]. " (Dodge, 1972 : 185)

Le second mot du glossaire, "matow" (ou matau), désigne le fer et par extension les objets forgés. Puis viennent les mousquets, leurs munitions, ainsi que des pièces de tissu (cloth) d'origine
Le porteur du titre Tui Bua entre dans la catégorie des Turaga définie plus haut. Le personnage décrit par Lockerby est présenté comme le chef le plus important de la région de Bua en 1808-1809, et comme un acteur incontournable du marché du bois de santal à Fidji grâce à l'intercession dont il est capable auprès de chefs dont l'autorité est plus localisée (Dodge 1972 : 185). Quain (1948 : IX-X) a en outre montré que, comme ailleurs au centre et à l'est de Fidji, les Turaga de Bua appartiennent à une " caste " à part en tant que descendants d'immigrants polynésiens (Tongiens). Il existe cependant des incertitudes sur l'autorité du chef sous la protection duquel Lockerby se trouve. En effet, le nom qu'il donne pour ce Turaga est "Beumbowala ", " Blambowalla » (Dodge, 1972 : 183, 187) ou "Beumbowallow" (Im Thurn et Wharton, 1925: 13-14). Dodge traduit cette transcription phonétique par "Buli Bouwalu» (1972: 183 n.7). C'est à dire qu'il s'agirait d'un titre plutôt que d'un nom, désignant le chef résidant à ( $\mathrm{Na}$ )Bouwalu, un village aujourd'hui situé dans le district de Vuya de la province de Bua. En raison de l'autorité étendue que Lockerby impute à son hôte, on peut penser qu'il s'agit bien du Tui Bua, c'est à dire du Turaga principal de la région, si tant est que le titre soit déjà en place avant 1810 ce qui n'est pas avéré. Lockerby se contredit cependant en affirmant, d'une part, que l'autorité du chef de «Myemboo Bay " [Mai Bua Bay] s'étend à l'île [de Vanua Levu] toute entière (Dodge, 1972 : 185) et, d'autre part, qu'il existe quatre chefs principaux sur cette île, dont Im Thurn et Wharton déduisent qu'il s'agit des Tui Bua, Tui Macuata, Tui Cakau et Tui Dama (1925 : 21 n.1). En effet, Lockerby décrit plusieurs chefs inféodés à son protecteur (Dodge, 1972 : 186-187), mais il n'y inclut pas celui qu'il nomme "Buledam ", Buli Dama, le chef du district de Dama (Im Thurn et Wharton, 1925 : 23-24 n.2). Ce dernier est présenté davantage comme un égal du protecteur de Lockerby que comme un de ses sujets (Idem : LXXIV, LXXVI). Ceci laisse deux possibilités. Soit l'hôte de Lockerby est le Tui Bua, et "Buledam » son voisin et homologue dans la chefferie voisine de Dama; soit on se situe au niveau de ce que l'on considère aujourd'hui comme des districts de la province de Bua, soit Bua, Vuya et Dama, sachant que ces subdivisions ont été généralisées, parfois schématiquement, au début de la colonisation britannique sans correspondre toujours à la réalité historique. En référence à Lockerby qui désigne Buli Bouwalu comme le chef de Bua, je parle ici de Tui Bua. Le lecteur est toutefois prié de garder à l'esprit l'ambiguïté qui existe, inhérente aux sources.

occidentale. Un certain nombre de denrées locales sont aussi listées, témoins des préoccupations de subsistance des marins et des transactions annexes au trafic du santal.

18. Les termes « roi » et « royaume » sont inappropriés à la géopolitique du xIx siècle à Fidji. Mais cette terminologie est fréquente dans la littérature.

19. Toutes les traductions sont de l'auteur. 
Plus loin, Lockerby décrit les chefs de Bua, leurs relations, ainsi que leur influence. Surtout, il indique leurs dispositions envers les Occidentaux (Dodge, 1972: 185-187), soulignant leur rôle de partenaires d'échange incontournables, notamment en ce qui concerne le chef principal de Bua. Il fournit ensuite une liste d'articles de troc utiles pour lesquels il suggère les quantités qu'il conviendrait d'apporter à Bua (idem: 187). Il met ainsi en lumière un certain nombre d'objets d'échange, importants à la charnière des années 1800-1810.

Encore une fois, l'ivoire figure en tête de liste. "Ivoire façonné en forme de dent de cachalot" arrive en première position, suivie de la mention " ivoire de toute sorte", puis de "couteaux avec un manche d'ivoire». Lockerby précise : « 400 livres d'ivoire donneront environ 2000 dents d'une livre (lb) à une once (os) chacune. » (Dodge, 1972: 187)

Lockerby recommande aussi l'emploi de certains coquillages blancs «en aussi grand nombre que possible ». Comme les dents de cachalot et leurs substituts, ces derniers montrent une prise en compte - à défaut peut-être d'une compréhension - des systèmes symboliques fidjiens. Dans l'imaginaire collectif local les dents de cachalot comme certains coquillages (par exemple buli vulalbuli dina (Ovula ovum) blancs et buli kula (Cypraea aurantium) de couleur jaune orangé) sont des matériaux prestigieux venus de la mer, et à ce titre l'apanage des Turaga (Hooper, 2013 : 103). C'est aussi le cas de leurs couleurs - blanche à brun rougeâtre - que l'on retrouve dans les attributs traditionnels du pouvoir comme les ornements pectoraux et les étoffes d'écorce battue portés par les chefs.

Dans sa liste des objets indispensables au marchandage santalier, Lockerby compte aussi des miroirs, et de petites pièces de tissu rouge "pour suspendre les dents [de cachalot]». Le rouge vif des tissus européens, et de certaines perles de verre, rappelle une couleur prestigieuse à Fidji. Rare dans la nature, elle est notamment associée aux plumes d'un perroquet endémique (kula). Il s'agit d'un rouge vif chatoyant qui peut avoir des reflets bleus ou verts, et dont les Fidjiens disent parfois en anglais qu'il est "doré " (golden). Les plumes rouges, les choses qui leur sont apparentées (tissus, perles de verre) et cette couleur " rouge doré " sont étroitement associées à ce qui est précieux voire sacré à Fidji, par exemple aux coquillages buli kula qui sont des insignes de rang, ou à l'île de Burotu - le royaume englouti des morts de la tradition polynésienne.

La mention de dents de cachalot entrelacées de tissu européen et d'une grande quantité d'ivoire en circulation est d'autant plus marquante ici que ces mêmes catégories sont absentes des collections muséales de la période. Cette non-représentation admet très peu d'exceptions jusqu'à la fin des années 1830 (Leclerc-Caffarel, 2013 : 127, 181). Ces biens ne circulent alors que dans un sens, des marchands vers les Turaga. Extrêmement précieux pour ces derniers, ils soulignent leur succès dans les transactions avec les Occidentaux et évoquent de plusieurs manières leur souveraineté (par exemple, origine marine, couleurs).

\section{Conflits et conceptions hiérarchiques à l'œuvre}

Les écrits de Lockerby donnent aussi à voir la place des conflits armés dans les premières relations entre chefs fidjiens et Euro-Américains. D'une part, les Occidentaux associés aux chefs qui leur procurent du bois de santal prennent part aux conflits locaux. D'autre part, l'exploitation du santal elle-même génère des querelles et des trahisons (Derrick, 1957 : 42-43). Ainsi, Lockerby explique avoir pris part à une attaque contre le Tui Bua - pourtant son protecteur - en échange de la promesse des rebelles de lui fournir du santal en octobre 1808 (Im Thurn et Wharton, 1925 : 46). Mais, le mois suivant, Lockerby et d'autres marins combattent à nouveau aux côtés du Tui Bua (idem : 53).

Ces exemples éclairent le rôle des marins étrangers dans l'équilibre politique local. Tour à tour ennemis ou alliés, ils sont aussi des objets de convoitise (ibid. : 54). À travers eux, c'est l'accès au marché lucratif du santal qui se joue. Les Européens deviennent eux-mêmes des monnaies d'échange, parfois troqués contre des biens d'origine occidentale. Ainsi, Lockerby explique comment, alors que le capitaine du General Wellesley sécurise une transaction de bois de santal en détenant des otages fidjiens de haut rang, il est lui-même en proie à la colère de leur peuple. C'est alors que :

"Bulendam [Buli Dama] and the father of one of the chiefs prisoners on board ransomed me and my comrade from the chief who had us in his possession for two whale's teeth, two tomahawks and two pieces of iron." (Im Thurn et Wharton, 1925: 47)

Enfin, Lockerby montre combien les Occidentaux se familiarisent rapidement avec les croyances et les pratiques religieuses fidjiennes, et vice versa. Il explique par exemple avoir évoqué « le dieu de l'homme blanc» à plusieurs reprises pour lutter contre des actes jugés barbares, comme la strangulation des veuves de haut rang (Im Thurn et Wharton, 1925: 63-65). Quant aux croyances fidjiennes, Lockerby témoigne d'une certaine compréhension de leur fonctionnement, au point de pouvoir identifier un cadeau de marque - tel le corps entier d'un homme " cuit de la manière qui est toujours employée lorsqu'ils font des présents à leur chefs principaux » (idem : 66-67) - et de les utiliser à son avantage : 
"On the 16 $6^{\text {th }}$ of May [1809] I went about ten miles up the river Embagaba to a village where I was told there was a large lot of Sandlewood; but the owners wanted a large whale's tooth for it, and I had not one to give. As the ship had almost completed her cargo, I wished very much to get this parcel. To accomplish my end I made use of a stratagem that answered the purpose. I told the native that the ship's Callow [Kalou $\left.{ }^{20}\right][$.... had sent me for the wood and ordered me to pay for it in ironwork. [...] I then took some long grass and bound it round several of his bread fruit trees; this done I made a pile of stones before the pond of fresh water which was before his door; and then told him whoever should eat of the bread fruit would die, and that the same fate would meet the person who should wash himself in the pond [...] After a good deal of persuasion I was prevailed upon to take off the taboo, and received a lot of wood as a present to the ship's Callow. It was soon carried down to the boat with more yams, plantains, orc. than it could contain [...] Afterwards I made them a present of a quantity of iron and beads, of far more value to them than a whale's tooth, if they could have understood their own interests. " (Im Thurn et Wharton, 1925: 65-66)

L'extrait ci-dessus ne prend en compte ni la valeur d'une "grosse dent de cachalot " ni le rôle stratégique d'une offrande au "Kalou» du General Wellesley pour les villageois. Il témoigne en revanche d'une certaine aisance de Lockerby parmi ses hôtes et tend à montrer que les habitants de la région perçoivent les visiteurs occidentaux selon une hiérarchie proche de la leur. Ainsi, les dieux/ esprits évoqués par le subrécargue, comme leur pouvoir de nuisance, semblent gérés à l'aune des pratiques fidjiennes traditionnelles. Par exemple, le chef de district refusant de passer outre la strangulation d'une femme de chef près de "Nandorey " Bay, en dépit d'une offre de "plusieurs dents de cachalot, de perles, \&c. ", craint d'abord les parents du défunt et les chefs alentour. Mais une fois la strangulation accomplie, il en vient à craindre le "Kalou de l'homme blanc", qu'il espère apaiser par un sacrifice de noix de coco (Im Thurn et Warthon, 1925 : 63-64) ${ }^{21}$.

Les capitaines des navires santaliers sont, eux, assimilés à des chefs. Ceci donne lieu à un certain nombre de mésinterprétations dans la littérature. On en déduit souvent qu'ils sont devenus des chefs locaux, ou qu'ils ont été a priori perçus comme tels (par exemple Campbell, 1980 ; Maude, 1964). Or, ils ne sont probablement jamais considérés comme des Turaga à Fidji ; mais puisqu'ils sont les interlocuteurs occidentaux les plus élevés en rang aux moments des transactions qui nous intéressent, ils ont pu être assimilés à des chefs étrangers par les Turaga, et notamment à leurs propres supérieurs hiérarchiques restés en Europe ou en Amérique (cf. Hooper, 1996 : 250, 264). Ce doit être le cas du Capitaine Scot [sic] qui reçoit en cadeau le corps humain dédié à la consommation cannibale déjà cité (Im Thurn et Warthon, 1925: 66-67). Scot n'en demeure pas moins un étranger (vulagi), traité comme un chef selon l'étiquette fidjienne.

L'exploitation du bois de santal à Bua pose les bases des relations entre Fidjiens et Occidentaux avant 1855. Les exemples ci-dessus montrent qu'elles reposent largement sur des échanges, dès les premières années du XIX ${ }^{e}$ siècle. De prime abord commerciales, ces transactions revêtent aussi un caractère politique. Elles influencent notamment le positionnement hiérarchique des chefs les uns par rapport aux autres. Certains aspects religieux (par exemple l'évocation du Kalou de l'homme blanc) sont manipulés par les Occidentaux pour orienter les échanges en leur faveur, alors que l'introduction d'armes à feu et de combattants européens, comme l'importation massive d'ivoire, impactent durablement la façon qu'ont les Turaga d'asseoir leur pouvoir par des succès guerriers et via l'accumulation et la circulation de biens importés de grande valeur. Sahlins (1983 : 90-91) note d'ailleurs une augmentation de la consommation cannibale, parallèle à l'accroissement de la quantité d'ivoire de cachalot en circulation à Fidji dans la première moitié du XIx ${ }^{\mathrm{e}}$ siècle, aussi symptomatique des luttes de pouvoir intenses qui s'exercent alors entre les chefferies. Ce qui précède montre surtout que les chefs de Bua, en particulier celui qui jouit de la plus grande autorité, sélectionnent les biens qu'on leur propose. Certains Occidentaux familiers des us locaux savent s'adapter à leurs demandes et au fonctionnement hiérarchique de la chefferie. Ainsi, des catégories symboliques et culturelles fidjiennes traditionnelles conditionnent les premières relations entre chefs fidjiens et marins occidentaux - aussi sûrement que les intentions occidentales sinon davantage. Progressivement, les possibilités d'obtenir du santal à Bua se réduisent - en raison de la surexploitation et d'un nombre accru de conflits entre marchands et indigènes (Derrick, 1957 : 42-43). À la recherche de trépangs, les marchands européens, américains et australiens délaissent alors Bua au profit d'autres régions de Fidji, septentrionales (Cakaudrove, Macuata) et centrales (Bau, Ovalau, Viwa).

20. Originellement, le terme kalou semble avoir désigné les esprits, ceux des morts et des ancêtres comme ceux de la nature. Par extension et sous l'influence des missionnaires, il renvoie aussi à ce que nous appelons « dieu " dans la tradition judéo-chrétienne (Capell, 1941 : 96-97).

21. À Fidji, les cocotiers et leurs produits (palmes, noix) sont considérés comme des offrandes venues de la terre (vanua) (Hooper, 2013 : 129). Ils sont aussi utilisés pour signifier et lever les prescriptions religieuses (tabu) (Leclerc-Caffarel, $2013: 104$ 105) ou bien encore pour les oracles (Henderson, 1831 : 39 ; Seemann, 1862 :377-378 ; Leclerc-Caffarel, 2013 : 175, 179-180). 


\section{Bau : la fabrique du pouvoir}

Souvent, la chefferie de Bau est considérée comme la plus importante de l'histoire fidjienne. Ceci tient notamment au rôle des Turaga de Bau dans le placement de l'archipel sous protectorat britannique en 1874 . La coopération voire l'initiative de Cakobau dans cette affaire sera discutée en fin d'article. Cakobau est le sixième Vunivalu de Bau. Installé en 1853, on lui attribue une influence croissante depuis la fin des années 1830. À partir de 1871, il est officiellement appelé Tui Viti (chef de toutes les îles Fidji). Pourtant, ni la prépondérance de Bau dans l'archipel ni celle des Vunivalu à Bau même ne sont avérées historiquement ${ }^{22}$. Plus que les enjeux de cette ascension, ce sont ses mécanismes qui m’intéressent ici, en particulier le rapport des chefs de Bau aux hommes, aux objets et aux idées venus d'Occident.

Le second porteur du titre de Vunivalu de Bau, Banuve, règne de 1770 à 1803 environ. De son époque, une seule référence explicite à la présence occidentale subsiste. Il s'agit de son nom posthume, Banuve Baleivavalagi ou «Banuve mort d'une maladie européenne $»^{23}$. Plusieurs historiens décrivent une épidémie de choléra asiatique (lila balavu) dans les premières années du $\mathrm{XIX}^{\mathrm{e}}$ siècle - suite au naufrage du schooner Argo.

\section{Naulivou et Charles Savage}

Le règne de Naulivou, fils et successeur de Banuve, est davantage marqué par la présence occidentale. Il reste en particulier associé au nom de Charles Savage. Ce personnage est l'objet d'une véritable légende, dont la construction au XIX et au Xx siècle a été analysée par Campbell (1980). Ce que l'on sait avec un certain degré de certitude, c'est que Savage arrive à Fidji via Tonga en 1808, à bord de l'Eliza qui s'échoue près de Nairai (mer de Koro). À Fidji, sa connaissance du tongien et du maniement des armes à feu se révèlent utiles; et il semble avoir été apprécié par Naulivou après son passage vers Bau à une date inconnue. Comme d'autres beachcombers, il est employé par le Turaga comme stratège militaire, et pour manipuler ses propres armes à feu. Il est récompensé en fonction de ses succès. Il meurt en 1813 à Wailea (Vanua Levu), dans une rixe entre marchands de bois de santal et indigènes (Dillon, 1829, I : 12-24).
Certaines sources, postérieures à sa mort, lui attribuent le titre "d'homme blanc du Vunivalu " (Tatawaqa, 1913) ou Koroi ni Vunivalu (guerrier du Vunivalu). Elles associent son nom à d'importantes victoires de Bau dans lesquelles l'armement occidental aurait permis de l'emporter, à Kasavu et à Nakelo notamment. Là où l'on mentionne son histoire, il est aussi souvent question de son mariage avec au moins une femme de haut rang, peut-être une fille du Roko Tui Bau appelée Kakua ou Kapua (Campbell, 1980).

Ce dernier point, comme l'attribution d'un nom honorifique et d'une réputation guerrière teintée de férocité, peut être comparé au traitement de certains combattants fidjiens indépendants, alliés au Vanua par les Turaga au XIX ${ }^{\mathrm{e}}$ siècle alors que le cannibalisme est encore avéré à Fidji. Sahlins (1983 : 83-84) décrit deux castes de guerriers étrangers au Vanua dont l'une des fonctions est de fournir au Turaga des victimes sacrificielles (bokola) pour la consommation cannibale. Les premiers sont des guerriers terriens, dits bati balavu par opposition aux bati leka qui appartiennent au Vanua. Les seconds sont des marins et des pêcheurs, des gens de la mer, kai wai. Sahlins explique comment, en plus de leur relation au Vanua, certains Turaga assoient leur pouvoir sur cette troisième catégorie, constituée "d'hommes dangereux" (tamata rerevaki). Leur partenariat est scellé et réitéré par un échange de femme(s), et dans le sens inverse de victimes sacrificielles. En particulier, les leaders des clans guerriers qui fournissent au Turaga des bokola lui seraient originellement "attachés " - et assujettis - "par le don d'une fille royale " (idem: 83-84). Entre Turaga et guerriers, la circulation des épouses et des victimes sacrificielles se fait donc à l'inverse de celle entre Turaga et Vanua. Initialement, c'est en effet le Vanua qui cède une femme de haut rang au Turaga en échange d'une part de sa divinité. Cette dernière est considérée comme bienfaisante une fois le Turaga - également étranger et dangereux - «domestiqué » comme un dieu local (Sahlins, 1985). Elle est synonyme d'une grande efficacité empreinte de surnaturel (mana). Dans la première moitié du XIX ${ }^{e}$ siècle, la nature divine du Turaga se manifeste notamment par sa consommation de chair humaine, une nourriture réservée aux dieux. Une partie de cette nourriture sacrée (tabu) peut être redistribuée au Vanua, comme un peu de la divinité et du mana auxquels

22. À l'origine, le porteur du titre Roko Tui Bau est le chef principal de Bau. Il est le leader spirituel par opposition au Vunivalu, considéré comme un chef de guerre. Au cours du XIX ${ }^{e}$ siècle - à partir du règne de Naulivou (Vunivalu) - les Vunivalu montent en puissance au détriment des Roko Tui Bau et la distinction des fonctions spirituelles et temporelles se fait moins sensible (Sahlins, 2004 : 63 ; Routledge, 1985 : 43 ; Toganivalu, s.d. ; Hocart, 1970 : 164).

23. Le terme valagi ou vavalagi vient du tongien palangi ou papalangi. Il désigne tout ce qui a trait à l'homme occidental, blanc, y compris son lieu d'origine virtuel au-delà des mers. Il se distingue du terme vulagi qui indique ceux qui ont le statut de visiteurs ou d'invités ; c'est-à-dire qu'ils sont temporairement présents dans la communauté. Culturellement, vulagi s'oppose à itaukei (autochtones) (Hooper, 1996 : 247). Valagi et vulagi diffèrent aussi du terme kai tani, qui renvoie à l'étranger au sens de celui qui est d'une autre nature. Par essence, le Turaga tel que le décrit Sahlins (1983; 1985) est un kai tani par rapport aux gens du Vanua. 
les autochtones ont droit grâce à leur association avec le Turaga (Sahlins, 1983).

Savage est un marin dont la réputation de guerrier s'est inscrite dans la tradition orale, quoique en grande partie après sa mort (Campbell, 1980). Il pourrait être comparé aux kai wai, qui font la force de Bau (Sahlins, 2004: 63-64). Cependant, de fortes différences entre le beachcomber et ces marins-guerriers subsistent. En particulier, il n'est nulle part question d'une fille de Naulivou lui-même, ou issue de son mataqali (Tui Kaba). De surcroît, si le mariage de Savage avec la fille du Roko Tui Bau était attesté, il convient de rappeler les tensions entre les porteurs des deux titres principaux de Bau (Vunivalu et Roko Tui). De fait, le Roko Tui est probablement en exil au moment d'un tel mariage (Toganivalu, s.d.). En poussant plus loin l'analyse, on peut même envisager ce mariage comme une humiliation infligée au Roko Tui Bau. En effet, il est de coutume à Fidji au début du $\mathrm{XIX}^{\mathrm{e}}$ siècle qu'un chef vaincu cède au vainqueur une de ses filles, en signe de reddition (soro). En refusant d'épouser lui-même cette fille, et plus encore en faisant en sorte qu'elle épouse Savage, Naulivou aurait pu marquer son opposition à toute réconciliation possible avec les membres du mataqali Vuaniivi, désormais privé du titre Roko Tui Bau au profit du clan Vusaratu (Toganivalu, s.d.). En d'autres termes, que cette rumeur de mariage avec une femme de haut rang ait été interprétée comme une prérogative octroyée à Savage indique surtout une projection des modèles occidentaux sur le cas fidjien par les observateurs historiques ${ }^{24}$. Finalement, la mention récurrente d'une opposition de Savage à certaines pratiques fidjiennes, dont le cannibalisme (Campbell, 1980), ne va pas non plus dans le sens de la comparaison avec les kai wai.

Ici encore, les Occidentaux semblent intégrés à la société fidjienne au gré d'enjeux politiques et selon des modalités qui vont au-delà des relations avec les Euro-Américains. Ces derniers forment une catégorie à part. Ils sont fondamentalement étrangers (kai tani-d'une autre nature) au système fidjien, même lorsqu'ils sont des hôtes (vulagi) acceptés voire appréciés. Les partenariats les plus pérennes s'établissent néanmoins au gré d'échanges (de services, de femmes, etc.), comme il est d'usage à Fidji avec des protagonistes qui n'appartiennent pas au Vanua.

\section{Le rôle des armes à feu}

Au-delà des controverses, le fait que le nom de Savage ait perduré dans la tradition fidjienne, associé à celui de Naulivou et aux armes à feu est intéressant. Plus que Savage et ses acolytes, il semble que les fusils firent de Naulivou un stratège redoutable (Routledge, 1985: 43). Après l'effondrement du marché du santal, les Occidentaux sont pour quelques temps moins nombreux à Fidji et l'apport en armes à feu est ralenti. Naulivou a donc le temps de consolider ses victoires grâce à celles déjà en sa possession (idem : 47). En dix ans environ, entre le milieu des années 1810 et le milieu des années 1820, il réussit à étendre son influence à la région centrale de l'archipel (mer de Koro) et à la petite île de Viwa, stratégiquement placée entre Verata ${ }^{25}$ et Bau. En s'appuyant sur les vasu de haut rang de $\mathrm{Bau}^{26}$ qui visitent régulièrement leurs parents maternels et collectent leurs tributs (Pauwels, 2015 : 160), il renforce aussi son autorité sur la côte Nord de Viti Levu, le sud de Vanua Levu, ainsi que sur les îles du centre (Lomaiviti) et de l'est (Lau). À la fin de son règne (1829), seule la chefferie de Rewa peut encore se prétendre l'égale de Bau (Routledge, 1985 : 48).

Progressivement, les mousquets qui font au moins en partie le succès de Naulivou deviennent une monnaie d'échange incontournable du trafic des bêches-de-mer. Ils semblent alors à peu près aussi valorisés que l'ivoire, et sont plus souvent que lui mentionnés dans les écrits des marchands. Ces derniers distinguent la pacotille - perles de verre et menus objets de métal - des objets de valeur qui viennent d'être cités (Leclerc-Caffarel, 2013 : 128). Avec les premiers, ils achètent de la nourriture et font de " petits cadeaux " (gifts). Livoire et les armes leur permettent d'acquérir les richesses qu'ils sont venus chercher à Fidji (par exemple trépang, écaille de tortue) et dans ce sens de faire des dons importants aux Turaga, les incitant au partenariat et à la réciprocité. A Bau en 1827, le capitaine Driver peut par exemple obtenir seize gros tonneaux pleins d'holothuries pour un mousquet. Il faut cinq à six jours de travail dans les récifs pour réunir cette quantité de mollusques ; et Driver est néanmoins perçu comme un homme généreux par les autochtones (Cary, 1998 : 64).

Toutes sortes d'armes à feu sont échangées contre des bêches-de-mer (Clunie, 2003b : 184 sq.) mais les fusils bon marché (flintlocks) sont les plus fréquents (Clunie, 1983a: 103). Clunie montre que les Fidjiens ne se contentent pas d'ajouter pistolets et autres fusils au répertoire des armes à leur disposition; ils les intègrent à des catégories préexistantes. S'ils avaient considéré les mousquets comme des objets d'emprunt, les Fidjiens auraient pu utiliser des consonances étrangères pour les désigner. C'est le cas d'autres

24. Mes plus sincères remerciements vont à Simonne Pauwels, pour son aide dans cette analyse du cas de Savage.

25. Verata est considérée comme une des chefferies de Fidji les plus importantes historiquement, son déclin en faveur de Bau est amorcé au début de la période qui nous occupe.

26. Cette stratégie est déjà celle de Verata, la chefferie dominant l'archipel au XvıII ${ }^{e}$ siècle (Routledge, $1985: 38$ ). 
biens importés, comme le cheptel bovin que l'on trouve parfois sur les navires occidentaux. En fidjien, le bovidé est appelé bulumakau (bull and cow) d'après les mots anglais de référence. Mais il n'y a rien à Fidji qui ressemble à une vache dans la faune endémique. Au contraire, les mousquets sont dits dakai, c'est-à-dire qu'ils sont appelés comme les arcs. Les balles sont nommées gasau (flèche/roseau). Cette terminologie montre une compréhension du fonctionnement des fusils, ainsi qu'une assimilation aux armes à projectiles fidjiennes traditionnelles ${ }^{27}$. De plus, ces dakai importés ressemblent à certaines massues fidjiennes comme les cali, munis de bords contondants redoutables (idem : 107). Ils apparaissent donc non seulement comme des arcs particulièrement performants, mais aussi comme une combinaison de deux des armes de combat fidjiennes les plus efficaces. Il existe d'ailleurs dans les collections muséales de la fin du XIX ${ }^{\mathrm{e}}$ siècle des fusils incrustés d'ivoire, comparables aux massues prestigieuses réservées aux chefs et aux guerriers de haut rang. Les plus fines de ces incrustations sont attribuées aux artisans polynésiens présents à Fidji pour y construire des pirogues, et qui fournissent aux Turaga des insignes de pouvoir au gré d'échanges attestés depuis la fin du XVIII ${ }^{\mathrm{e}}$ siècle entre Fidji et Tonga (Clunie, 2003a : 91 ; Kaeppler, 1978).

\section{La politique étrangère de Tanoa}

Si le règne de Naulivou est celui de la montée en puissance de Bau et des Vunivalu (Routledge, 1985 ; Toganivalu, s.d.), celui de son jeune frère Tanoa (1829-1852) est marqué par les dissensions internes et les crises politiques. En 1832, Tanoa est notamment victime d'un complot fomenté par ses demi-frères, Caucau et Ramudra - fils de Banuve et de sa quatrième épouse, Dabuli ${ }^{28}$. Les rebelles échouent à prendre la vie de Tanoa ${ }^{29}$, mais ils ont raison de celle de son héritier (Tubuanakoro) et de son trône. Tanoa le regagne en 1837 avec l'aide de son jeune fils, Seru (Cakobau). Après quelques années d'apaisement, un conflit oppose le Vunivalu au Tui Cakau de Somosomo. Finalement, une guerre éclate entre Bau et Rewa, pour durer de 1843 à $1855^{30}$.

En dépit - ou peut-être en raison - de l'adversité rencontrée dans les affaires internes, Tanoa prolonge la stratégie de Naulivou quant aux Occidentaux. Il s'évertue à en faire des alliés plutôt que des ennemis, et à canaliser leurs forces potentiellement destructrices à son avantage. Deux exemples illustrent cette ten- dance : le cas de David Whippy et la prise de position du Vunivalu dans le règlement de "l'affaire Bureau ».

Si Savage est "l'homme blanc" de Naulivou, Tanoa peut s'appuyer sur les talents de David Whippy pour mener à bien sa politique auprès des Occidentaux. Ce marchand américain - à Fidji depuis le début des années 1820 - est un fin négociateur et un bon interprète (Wilkes, 1845, III : 49, 58-59, etc. ; Cary, 1998 ; Wallis, 1851). Ces caractéristiques, la confiance que lui accordent les visiteurs occidentaux comme le Vunivalu de Bau, et la bonne connaissance qu'il a des îles en font un ambassadeur idéal. C'est à ce titre qu'il sert le Vunivalu en tant que matakibau (ambassadeur de Bau), puis le gouvernement américain comme agent commercial, avec une dimension consulaire de 1840 à 1846, avant la nomination de J.B. Williams (Campbell, 1998 : 66 ; Derrick, 1957 : 67 ; Wilkes, 1845).

Puisque le port de Levuka (Ovalau) offre de meilleures possibilités de mouillage aux navires euro-américains que Bau, Tanoa y transfere Whippy et demande au Tui Levuka (le Turaga d'Ovalau tributaire de Bau) de lui fournir une épouse (Toganivalu, s.d.) - selon le procédé déjà évoqué de l'intégration des étrangers au Vanua. Malheureusement, Toganivalu ne précise pas si l'épouse choisie est une fille du Tui Levuka, ni même si ce mariage a réellement eu lieu. L'histoire montre néanmoins le caractère décisif de ce transfert. Le settlement occidental à Levuka est voué à devenir la première capitale coloniale du groupe.

De façon générale, Whippy semble avoir influencé l'avenir de Fidji en favorisant et en accompagnant la transformation de la présence euro-américaine dans l'archipel. Il se positionne par exemple en faveur des missionnaires, et - fait rare - semble avoir réussi le passage du statut de beachcomber à celui de colon (settler). Il est à ce titre précurseur dans un certain nombre d'entreprises occidentales à Fidji, dès les années 1840. Ainsi, au commerce de bêches-de-mer, il faut ajouter la construction navale à l'occidentale, la plantation de canne à sucre et la spéculation foncière. Finalement, il semble être passé d'une profonde adaptation au monde fidjien autochtone, décrite par Cary (1998) dans les années 1820 , à une collaboration plus autonome avec les Turaga, engendrant des désaccords avec Cakobau (Campbell, 1998 : 62-68).

"L'affaire Bureau » est un autre exemple de l'impact de la politique étrangère de Tanoa sur l'équilibre des forces de la région. De prime abord, les Européens impliqués dans cet incident semblent

27. De la même façon, on a vu qu'en 1813 Lockerby utilise "matow " (i.e. matau) pour désigner les outils en fer. C'està-dire qu'il les appelle comme la lame de l'herminette traditionnelle. Cette homonymie souligne un usage antérieur que les pièces métalliques obtenues des Euro-Américains - dont des lames de haches - viennent remplir.

28. Naulivou et Tanoa sont les fils de Lewasaki (ou Lewasau), l'épouse principale de Banuve, originaire de Rewa.

29. Tanoa fuit à Somosomo sous la protection du Tui Cakau, alors allié à Bau (Toganivalu s.d.).

30. Cet épisode de l'histoire fidjienne est largement discuté dans la littérature (Sahlins, 2004 ; Routledge, 1985 : 68-88 ; Derrick, 1957 : 85ff; Toganivalu, s.d.). 
avoir agi en toute indépendance. Mais une analyse plus poussée éclaire les enjeux locaux de la manœuvre.

Présent à Fidji au début des années 1830, le capitaine Bureau est un personnage assez obscur de l'histoire fidjienne, de nationalité française (Broc, 2003 : 98) - probablement un marchand d'holothuries et d'écaille de tortue et à ce titre aussi un trafiquant d'armes. Comme ses confrères santaliers, il semble qu'il ait eu l'habitude de prendre part aux conflits locaux, notamment auprès des rebelles de Bau, Mara et Seru Tanoa (Routledge, 1985: 61). Cette attitude cause sa mort en 1834, au large de Viwa.

Lorsque Dumont d'Urville revient à Fidji en octobre 1838 à la tête des corvettes Astrolabe et Zélée pour compléter ses relevés cartographiques $^{31}$, il a aussi pour mission de faire respecter le nom de la France dans les territoires visités. C'est pourquoi il décide de mener une expédition punitive contre les responsables présumés du meurtre de Bureau. D'après les renseignements obtenus de sources plus ou moins concordantes - certaines proches du Vunivalu de Bau - il faut blâmer Namosimalua (Tui Viwa) et son neveu Verani (Dumont d'Urville, 184154, IV : 171-172, 179-181, 201). Le 17 octobre 1838, une troupe d'hommes armés incendie le village de "Piva " (Viwa) avant de le mettre à sac. Malheureusement pour d'Urville, Namosimalua et ses hommes, prévenus, ont fui avec leurs effets personnels ne laissant qu'un maigre butin aux militaires français. G. de Rocquemaurel, le second de d'Urville sur L'Astrolabe, raconte la scène dans son journal (1837-40) :

«Les flammes, chassées par le vent, nous forcèrent à la retraite, et nous quittâmes au plus vite la petite baie pour venir attaquer l'autre partie du village. Mais la disparition de l'ennemi a déjà rendu notre expédition plutôt vandale que militaire [...] Le village fut occupé par nos gens tandis que les hauteurs étaient gardées par quelques hommes, on visita toutes les cases qui ne fournirent qu'un butin assez mince. Des nattes en lambeaux, quelques ustensiles [de] ménage, des pots en terre cuite, les premiers que nous eussions [vus] dans l'Océanie, quelques corbeilles à provisions assez bien tressées composèrent tout le butin des maraudeurs. Les naturels avaient emportés leurs armes, leurs étoffes et leurs provisions. "

Malgré cette maigre récolte, d'Urville est satisfait (1841-54, IV : 201). Il ignore qu'il a été l'instrument de la vengeance de Tanoa contre son versatile vassal, et que comme Bureau il a engagé ses hommes dans les conflits locaux. En 1838, en effet, l'assise de Tanoa sur le trône de Bau est précaire (Calvert, 2003 : 21 ; Wilkes, 1845, III : 17). L'expédition punitive contre Namosimalua, qui s'est associé aux rebelles de

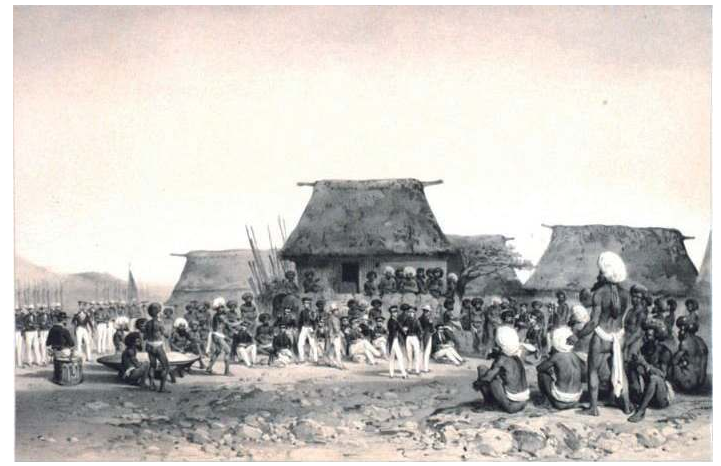

Figure 1. - «Réception des Français à Pao" (Dumont d'Urville, 1841-54, Atlas : pl. 83)

Bau avant de vouer de nouveau allégeance au Vunivalu, va dans le sens de l'autorité retrouvée. Tanoa accorde son aide pleine et entière à d'Urville (Dumont d'Urville, 1841-54, IV : 193), au point d'abriter les corvettes dans la baie de Bau pendant l'attaque. Après la "victoire", il accueille les officiers français en grande pompe, leur offrant yagona (kava) et magiti (banquet) comme à des alliés (idem : 204-210).

En plus d'avoir de facto renforcé l'autorité de Tanoa, l'événement bénéficie aux missionnaires. Abattu par sa défaite, le Tui Viwa se met en quête de nouveaux partenaires. Peu de temps après l'incendie de Viwa, il demande aux méthodistes présents à Fidji depuis 1835 de lui envoyer un indigène converti pour lui enseigner le lotu (christianisme). Bientôt, Namosimalua se déclare en faveur du lotu, quoiqu'il ne se convertisse jamais officiellement et refuse de renoncer aux pratiques qui font horreur aux missionnaires - dont le cannibalisme et la polygamie (Faure, 1929 : 36). Malgré cette réserve, Calvert considère le soutien du Tui Viwa au lotu comme une avancée décisive. Il rend possible, dit-il, l'installation de chrétiens à Viwa - stratégiquement positionnée vis-à-vis de Bau (Calvert, 2003 : 283). C'est là aussi que de premières conversions significatives ont lieu. En 1844, Vatea se convertit (idem: 276-277). Elle est l'épouse de Namosimalua et une sœur classificatoire de Cakobau - la fille de Caucau, un chef rebelle vaincu (voir plus haut). En 1845, c'est Verani, le neveu de Namosimalua et un fidèle conseiller de Cakobau qui choisit la voie du lotu, affectant le futur Vunivalu par sa décision (ibid. : 263 sqq. ; Wallis, 1851 : 65-66).

\section{Les insignes du pouvoir}

Progressivement, Tanoa et son ambassadeur Whippy deviennent des interlocuteurs incontournables pour les Occidentaux (Campbell, 1998 : 64). En 1840, alors que Tanoa s'apprête 
à rendre visite au commandant de l'U.S. Exploring Expedition qui souhaite lui faire signer des accords pour la protection des bateaux de commerce américains, Wilkes décrit :

"Tanoa despatched David Whippy on board to inform me of his arrival, when I immediately sent Lieutenant Carr to call upon him and inform him that my boat would be at the shore in the morning for him [...]. He [Tanoa] was, after the fashion of his group, almost naked, having a small maro passed around his loins, with long ends to it, and a large turban of tapa cloth in folds about his head, so as almost to hide the expression of his countenance; his face was bedaubed with oil and ivory-black, as were also his long beard and mustaches [...] From his begrimed look he has obtained the sobriquet of "Old Snuff", among the whites; he is about sixty-five years old, tall, slender, and rather bent by age; on his breast, hanging from his neck, he wore an ornament made of mother-of-pearl, tortoise-shell, and ivory, not very neatly put together, and as large as a dinner-plate, (called diva ndina [civa dina]); on his arms he had shell armlets, (called ygato [qato]) made of the trochus-shell by grinding them down to the form of rings [...]." (Wilkes, 1845, III : 58)

Outre le rôle clé du Vunivalu dans les relations entre Fidjiens et Occidentaux et les modalités de ce qui paraît être une rencontre entre deux personnes d'autorité (Tanoa et Wilkes), cet extrait met en lumière l'ornementation corporelle du Turaga. En association avec d'autres attributs de son rang, Tanoa porte notamment un large pectoral composite. La description s'accompagne de la gravure ci-dessous ${ }^{32}$. En plus de l'ivoire de cachalot, importé, ce pectoral réunit des matériaux clés du monde symbolique fidjien. L'huître perlière (civa) est un marqueur de rang important dans toute la Polynésie (Clunie, 2003a : 82, fig. 100, 2013: 167). Les premiers ornements fidjiens de type civa dans la littérature sont mentionnés par Bligh en 1792 (Henderson, 1933: 152-153). Ils sont alors composés d'une simple coquille polie et sont inaccessibles aux voyageurs européens (Leclerc-Caffarel, 2013 : 59-61). Le civa porté par Tanoa est lui serti d'ivoire. Il inclut aussi un petit triangle d'écaille de tortue dans sa partie supérieure gauche (ci-contre, fig. 2).

Si le terme civa désigne originellement l'huître perlière (Pinctada margaritifera), il renvoie aussi aux pectoraux ayant la forme du coquillage mais réalisés avec d'autres matériaux. Par exemple, les ornements de nacre et d'ivoire sont dits civavonovono (civa incrusté). Civatabua désigne un assemblage de pièces uniquement en ivoire (Leclerc-Caffarel, 2013 : 119-124). Une fois encore, il semble qu'un matériau importé, l'ivoire, vienne remplacer, compléter ou renforcer une matière plus familière - la nacre d'huître perlière ${ }^{33}$.

Déjà évoqués par Warren Osborn en 1834, les civavonovono sont inaliénables ${ }^{34}$ en 1840 , alors même que les pectoraux les plus simples sont devenus accessibles aux Occidentaux ${ }^{35}$ :

"Pearl shells with the cross taken from their back, are worn by them [Fijians], some of these they consider very valuable, as they have been handed down from father to son for many generations. Old Snuff [Tanoa] has one which has been handed down in this way, it is broken and lashed together in many places, he always wears it upon his neck and takes great care of it. " (Osborn, 1833-35)

Hooper définit de tels ornements comme des regalia, des attributs du pouvoir des Turaga (2013 : 147-151). De facture vraisemblablement tongienne (Clunie, 2003a : fig.23, 117, 118 ; Damm, 1975), ils réunissent des matériaux précieux, pour certains emblématiques des relations commerciales avec les Occidentaux (ivoire ${ }^{36}$, écaille de tortue, parfois métal). Un tel plastron met en valeur le pouvoir intrinsèque du Vunivalu en tant que Turaga (c'est-à-dire un étranger venu de la mer, connecté à la Polynésie), ainsi que le succès de ses partenariats avec les Européens et la force de son réseau de relations dans la région.

Ce qui précède a montré comment des objets (dents de cachalot, fusils) et des hommes (Savage, Whippy) véhiculés par les Occidentaux deviennent des atouts politiques des Vunivalu

32. Dans les années 1980, cette image permet à Clunie (1983b) d'identifier le plastron en question dans les collections du musée d'archéologie et d'anthropologie de l'université de Cambridge (Herle et Carreau, $2013: 4$ fig.1.4, 73 fig.5.1, 82, 95-96), plus de cent ans après qu'il a été offert au premier gouverneur des Fidji (1875-1880), A. Gordon, par Cakobau.

33. Il est d'ailleurs probable que même les tabua, aujourd'hui invariablement en ivoire de cachalot, aient pu être autrefois faits d'autres matériaux (pierre, bois, coquillage, dent de morse) (cf. Deane, 1921 : 77-78 ; Brewster, 1937 : 40 ; Roth, 1973 : 97-99 ; Reid, 1990 : 10 n40, 20-21 ; Clunie, 2013, 2003a : 124 ; Hooper, 2013 : 146-147).

34. On sait qu'il existe traditionnellement à Fidji des objets "de famille " qui contrairement aux iyau précieux mais échangeables sont soigneusement gardés et transmis au sein des familles de haut rang. C'est le cas par exemple de ce que Hooper nomme les « heirloom tabua" $(2013: 141-145)$.

35. Le lieutenant Reynolds décrit « des ornements pectoraux faits [d'une seule pièce] de nacre, mais auxquels ils [Fidjiens] n'accordent pas une grande importance" (Hoffman Cleaver \& Stann, 1998 : 160-161). Plusieurs spécimens de ce type sont d'ailleurs présents dans la collection Wilkes conservée à Washington par la Smithsonian Institution (par exemple E3039-E3043, E4542). La concurrence des ornements composites semble avoir entraîné une dévaluation des pièces uniquement faites de nacre.

36. Les Fidjiens ne chassent pas la baleine. Les réseaux d'échange traditionnels entre les îles du groupe et au-delà permettent la circulation d'ivoire et d'os de cétacé, ainsi que d'objets manufacturés, avant l'arrivée des marchands occidentaux. Ces derniers changent néanmoins la donne par les quantités qu’ils importent à Fidji, entraînant une certaine démocratisation de l'ivoire. Les savoir-faire les plus perfectionnés sont attribués aux artisans tongiens et samoans (Hooper, 2013 : 129-133; $1982: 85-89)$. 


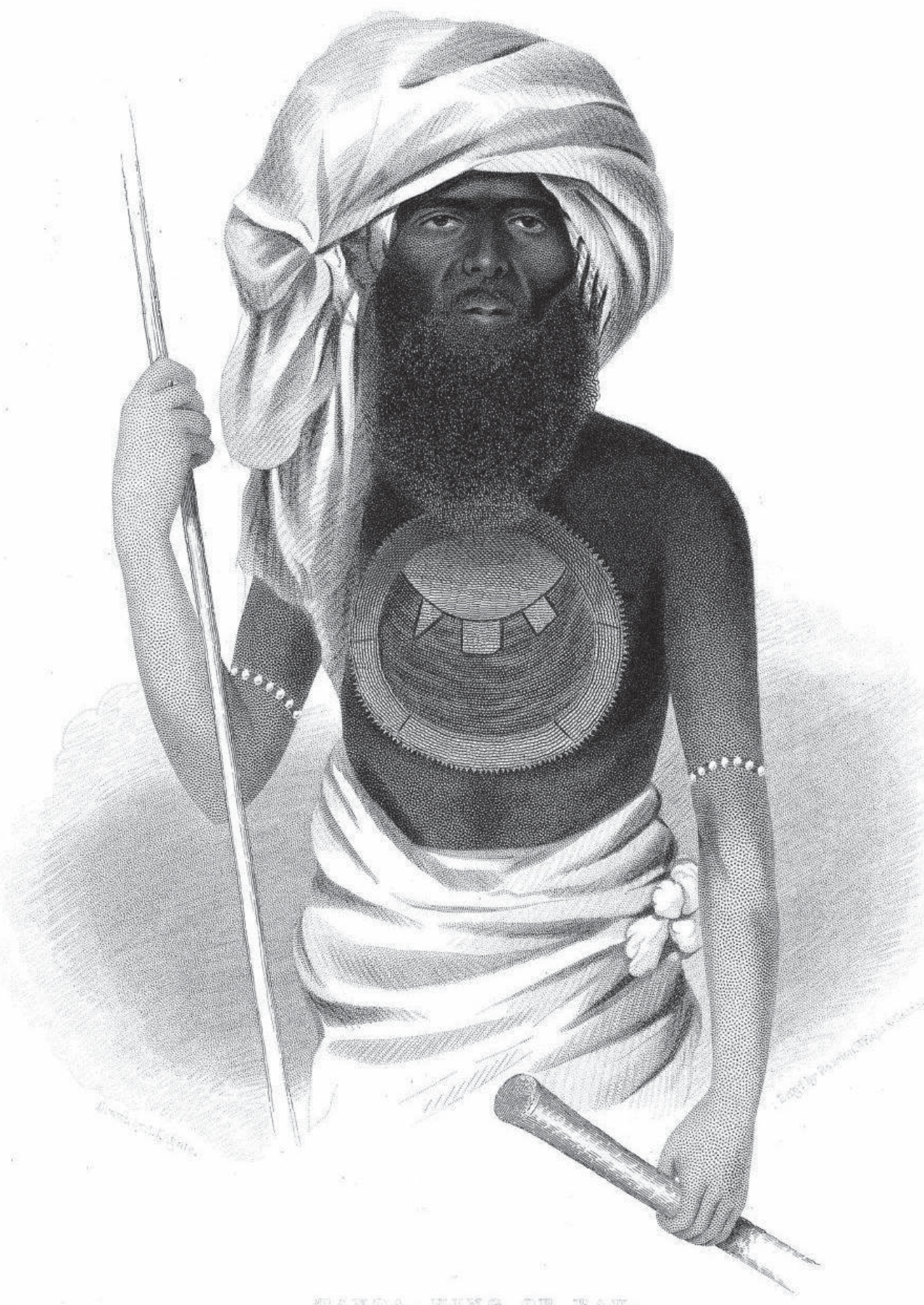

Figure 2. - "Tanoa" par A.T. Agate (Wilkes, 1845, III : 58)

de Bau avant 1855. C'est par l'échange d'objets de valeur, de prestations (banquets), de femmes (épouses) et de services (protection, médiation) que ces associations sont instaurées et maintenues. C'est-à-dire qu'elles le sont à la manière fidjienne. Comme en première partie, on constate une incorporation d'éléments extérieurs dans la sphère locale. En particulier, les marins euro-américains (beachcombers, marchands et officiers de marine) sont accueillis selon leur rang supposé au sein de la société occidentale qu'ils incarnent. Les services rendus et le potentiel qu' une alliance avec eux (commerciale, guerrière, etc.) représente sont aussi des facteurs importants. Ils sont donc traités selon des catégories qui combinent des représentations fidjiennes et européennes du pouvoir, en lien avec les différentes fonctions qu'ils occupent de facto auprès des Turaga (par exemple kai wai, mataki). Ils conservent néanmoins toujours leur statut d'étrangers (kai tani) et d'hôtes (vulagi). 


\section{Lau : le paramètre missionnaire}

\section{Un système économique spécifique}

De par leur situation géographique et le peu de ressources commerciales (trépang, santal) qu'elles offrent aux visiteurs occidentaux, les Fidji orientales (Lau) sont en retrait des flux d'échanges évoqués précédemment. Mais on trouve d'autres richesses dans ces îles; notamment un bois dur et sacré très valorisé, le vesi (Intsia bijuga), utilisé dans la fabrication des bols à kava de type polynésien (tanoa) et pour les grandes pirogues à double coque (drua) qui requièrent la combinaison de savoir-faire tongiens, samoans et fidjiens pour voir le jour (Young, 1982 : 35-36 ; Clunie, 2003a : 15, 21-22). En outre, la position et l'étendue des Lau, de Vanua Balavu ${ }^{37}$ à Onoi-Lau, en font un point de passage obligé pour de nombreux bateaux en provenance de Tonga, d'Australie et de Nouvelle-Zélande. Un grand nombre d'objets d'échange, locaux et importés, transitent donc par ces îles. Les Turaga de Lakeba (île principale) jouissent, eux, d'une position stratégique entre les chefferies de Somosomo, Bau et Tonga auxquelles ils sont connectés par intermariages (Young, 1982 : 32).

En conséquence, le contact direct avec les EuroAméricains semble subsidiaire dans l'approvisionnement de Lakeba en biens importés. Part belle est faite, en revanche, aux circuits d'échanges traditionnels qui opèrent dans les Lau et au-delà (Young, 1982: 35), en particulier via Tonga à l'est, Vanua Levu au nord et Viti Levu à l'ouest. Ceci pourrait expliquer certaines attitudes des Tui Nayau $^{38}$, qui semblent moins préoccupés de leurs relations directes avec les Occidentaux que de ce qu'elles impliquent en termes de politique locale. Ils sont probablement aussi refroidis par une mauvaise expérience suite au naufrage de l'Argo, en 1800 (Pauwels, com. pers.). Ceci étant dit, à la fois soucieux d'une bonne entente avec leurs alliés et suzerains et de l'allégeance des territoires sous leur autorité, les Turaga de Lakeba adaptent sensiblement leur politique vis-à-vis des Européens entre 1800 et 1855. L'évolution des rapports entre missionnaires et Tui Nayau est un bon baromètre de cette tendance. En voici un résumé. En 1830, le Tui Nayau Malani accueille froidement les missionnaires tahitiens qui lui sont envoyés par la London Missionary Society (LMS). En 1835, Taliai Tupou, le demi-frère de Malani devenu Tui Nayau à son tour, accepte que les révérends wesleyens Cargill et Cross s'installent à Lakeba ${ }^{39}$. En 1838, Hunt, Jaggar et Calvert se joignent à eux. Fidji devient alors un district indépendant de celui de Tonga pour les wesleyens (Calvert, $2003: 27$ ), et Lakeba le point de départ de leur expansion à travers l'archipel. En 1839, une chapelle est construite à Tubou (Lakeba). En 1842, une autre est édifiée à Oneata où des conversions ont lieu avec l'accord du Tui Nayau (idem : 93-94). En 1849, enfin, Taliai Tupou professe publiquement son rattachement à la religion chrétienne (ibid. : 136) - près de cinq ans avant la conversion de Cakobau.

La progression des Tui Nayau sur le chemin du lotu (christianisme) semble explicite. La compréhension des tenants et aboutissants de cette chronologie méritent néanmoins quelques approfondissements.

\section{Chronologie d'une conversion}

Premiers temps : " tolérance sans enthousiasme "

Malani et son jeune frère, Soroaqali, sont décrits par Young (1982) comme réfractaires aux incursions européennes dans les affaires des Lau. D'après Thornley (2005: 27), c'est pourtant Malani qui envoie Isereli Takai - un jeune Fidjien d'origine tongienne apparenté au Tui Nayau - vers l'est (jusqu'à Tahiti via Tonga) pour qu'il s'y renseigne sur la religion chrétienne. Quelques années plus tard, Malani règne encore quand Takai et ses acolytes tahitiens de la LMS (Arue, Hatai et Jacaro) se présentent à Lakeba. Ils s'installent d'abord à l'écart de Tubou, dans un endroit sans village appelé Tulaki (S. Pauwels, communication personnelle). Mais, en 1832 ils quittent Lakeba sans y avoir provoqué de conversions. Ils s'installent à Oneata, une île à laquelle Takai est apparenté. C'est là qu'ils commencent à faire des émules (Young, 1982: 43-44). Dans le même temps Ono-i-Lau, à l'extrême sud, embrasse aussi le lotu (idem : 43), et avec un certain militantisme en dépit des persécutions menées par Lakeba si l'on en croit les récits missionnaires (Calvert, 2003 : 51-90). Young explique ces conversions par « l'enthousiasme pour l'innovation" des îles périphériques $(1982: 32)$. Elles seraient mieux disposées que Lakeba au contact avec les Occidentaux, car relativement exclues du système économique mentionné plus haut (idem : 41-42). Elles sont aussi plus souvent fréquentées par des navires européens que le reste des Lau et indépendamment connectées à certains alliés de Lakeba comme Bau et Tonga (ibid. : 46).

En plus de devoir maintenir son ascendant sur ces "franges turbulentes" (Young, 1982: 46), le Tui Nayau doit veiller à satisfaire des alliés dont les intérêts divergent. Parmi ces derniers,

37. Quoiqu'elle fasse géographiquement partie des Lau, au début du XIX ${ }^{e}$ siècle et jusqu'aux victoires de Ma’afu dans les années 1850, Vanua Balavu est sous l'influence de la chefferie de Somosomo. Ma’afu est l’héritier de la lignée royale des Tu’i Kanokupolu (Tonga), et un acteur clé de l'histoire fidjienne (voir Routledge, 1985 : 76).

38. Pendant la période qui nous occupe, le porteur du titre Tui Nayau est le chef principal des Lau. Il est basé à Tubou (Lakeba). 39. Les wesleyens appartiennent à une autre branche de l'Église méthodiste. Ils se distinguent des missionnaires de la LMS. 
les Turaga de Lakeba sont liés par mariage aux Vunivalu de Bau depuis la fin du XviII ${ }^{\mathrm{e}}$ siècle au moins (idem: 40); et ils sont aussi unis aux iSokula de Somosomo ${ }^{40}$. Les chefs tongiens sont également nombreux à visiter les $\mathrm{Lau}^{41}$. Malani et Taliai Tupou sont eux-mêmes vasu de Tonga, et à ce titre peuvent faire appel à des guerriers tongiens pour mener leurs combats ${ }^{42}$ (Pauwels, 2015 : 158). Ces partenariats font en partie la fortune des Lau, mais ils sont parfois difficiles à gérer. Ainsi, en 1841 la guerre entre Somosomo et Bau met Taliai Tupou dans l'embarras, " obligé de choisir entre deux façons de se faire un ennemi redoutable " parmi ses alliés de longue date (Lyth in Young, 1982 : 47).

L'accueil des missionnaires chrétiens et la conversion de certains de ses vassaux posent encore davantage de problèmes au Tui Nayau. Bau est encore fermement opposé au lotu, dans le même temps supporté par un grand nombre de Tongiens (Young, 1982: 47-50). Aussi longtemps que possible Taliai Tupou mène ce que Young qualifie de "politique prudente, de tolérance sans enthousiasme". Mais pressé de toutes parts de prendre position, il avance vers la conversion.

En 1835, deux ans après avoir succédé à Malani, Taliai Tupou accède à la demande des wesleyens de s'installer à Lakeba. Ces derniers bénéficient du soutien tongien et d'une solide préparation (Henderson, 1931 : 72, 79; Schütz, 1977 : 60-61, 64 ; Calvert, $2003: 6$ ). Ces missionnaires se révèlent des partenaires d'échange importants, y compris dans l'approvisionnement des autochtones en objets d'importation comme des dents de cachalot, des articles de quincaillerie et des pièces de vêtement (Leclerc-Caffarel, 2013 : 255-259). Une fois à Lakeba, ils bénéficient de la protection du Turaga, comme il est d'usage à Fidji lorsqu'il est question d'étrangers dont la présence est acceptée (vulagi). Malgré cela, Cargill mentionne de médiocres succès en 1837 . Schütz résume ses propos :

"Tui Nayau continued to be vague. During a severe illness, he refused to participate in the customary ceremonies, but, on the other hand, made no positive move toward accepting the new religion. He said, "When Tanoa becomes a Christian, I will follow him”. " (Schütz, 1977 : 98)

Cette dernière phrase attribuée au Tui Nayau, comme les arrangements pris durant sa maladie, montrent que le Turaga a surtout à cœur de ne froisser aucun de ses alliés, ni ceux qui le sont de longue date (Bau et Tonga) ni les nouveaux (missionnaires). Les relations et la hiérarchie entre les chefferies conditionnent la progression du lotu. D'autres réticences de ce type sont mentionnées dans le journal de Cargill (Schütz, 1977 : 123, 169). La plupart désignent le Vunivalu de Bau comme le décisionnaire de l'avenir religieux de Fidji, et en font une cible clé des missionnaires.

\section{Deuxième temps : alliances changeantes}

En 1839 pourtant, avec l'accord de plusieurs Turaga, les wesleyens s'installent dans d'autres parties de l'archipel, notamment à Rewa, Somosomo et $\operatorname{Viwa}^{43}$. A partir de là, leur nombre ne cesse de croître, bientôt renforcé par d'autres missionnaires dont un grand nombre de Tongiens. La même année, ce sont les Tongiens résidant à Tubou qui y font construire une chapelle (Calvert, 2003 : 91).

En 1842, le cas d'Oneata marque un changement dans l'attitude du Tui Nayau. Taliai Tupou autorise plusieurs conversions, alors qu'elles se faisaient jusque-là sans son consentement. Mais, il est toujours réticent à suivre lui-même cette voie. Il semble craindre pour son autorité, notamment pour sa capacité à accumuler les biens nécessaires à sa fonction et à sa légitimité :

"September $27^{\text {th }}-$ The King and several of his attendants paid us a visit [...] we had a long discourse on the subject of religion. He seems to think the time for embracing religion is nigh; but fears lest the people will not obey him in that they will be slack in giving him property. " (Henderson, 1931: 105)

\section{Et Henderson d'ajouter :}

"That is, pay their taxes. This fear was well founded. By embracing Christianity and thereby abandoning his old gods from whom his authority was principally derived, the chief would be cutting the ground from under his own feet. " (Henderson, 1931 : 105 n81)

En 1844, la mort de Soroaqali change la donne. Il est le frère aîné de Taliai Tupou et l'un des plus fervents opposants du lotu dans les Lau. Si l'ordre traditionnel avait été respecté, Soroaqali aurait succédé à Malani. Mais son statut de vasu tau$k e i$, puisque sa mère est issue de la noblesse de Lakeba (mataqali Narewadamu), lui octroie une place suffisamment importante - plus importante

40. Vers 1837, Taliai Tupou a notamment épousé Qativi, une fille de Tuỉila’ila, futur Tui Cakau.

41. Rappelons que des artisans tongiens viennent dans les Lau pour y construire de grandes pirogues (Young, 1982 : 3536). De cette présence tongienne résultent un certain nombre d'échanges d'objets manufacturés, mais aussi de techniques et de motifs stylistiques. Les artisans tongiens fournissent notamment aux Turaga des objets de parure et de prestige en ivoire de cachalot. En outre, les sœurs des Tủi Tonga étaient autrefois préférablement mariées à des hommes fidjiens de haut rang (Kaeppler, 1978 : 247-248). Cette pratique n’a pas été perpétuée par les Vuanirewa (mataqali porteur du titre Tui Nayau pendant la période qui nous occupe), mais elle donne une idée de la profondeur historique des relations entre Fidji et Tonga.

42. Leurs mères sont toutes deux apparentées aux clans royaux Tüi Tonga et Tüi Kanokupolu.

43. Thornley donne des éléments de compréhension sur ce début de propagation du lotu, consenti par les Turaga (par exemple Thornley, 2000, 2002, 2005). 
même que celle de Tui Nayau - pour qu'il la préfère à celle de chef régnant. Il cède donc la position à son cadet, et promet d'assurer sa protection (Pauwels, 2015: 158). Il est un allié irremplaçable de Taliai Tupou, qui ne peut se permettre de l'offenser par sa conversion. Deux ans plus tard, le fils aîné de Malani, Wetasau (ou Vueta$\mathrm{sau}^{44}$ ), se convertit (Calvert, 2003 : 129). Puis, le 19 octobre 1849, Taliai Tupou professe publiquement son rattachement au lotu (idem: 136). Le 29 octobre, le révérend Lyth transmet la nouvelle à ses confrères :

"I have unusual things to communicate both pleasing and otherwise. Tuinayau \& his heathen friends have lotued. He has been at our Chapel two successive Sundays. He thuvaed [cuva, i.e. bow the head (Capell, 1941 : 46)] on Friday $19^{\text {th }}$ inst. and with him Bolabitu \& Veikoso - on the following day Tukana the priest \& $r$, on the Sabbath Tuitubou, \& since then the two Mata-ki-Baus. They have louted well \& stand firm. The old king has acted nobly - he at once determined on a meeting of the people to put the affairs of the land in order and accordingly assembled them on Thursday $26^{\text {th }}$. We were requested to attend [...] Lua, Wetasan 8 the other chiefs; and the representatives from all the towns attended. The meeting had reference to the management of the affairs of the land exclusively and was a very satisfactory one. Mara was expected but whether he would come peaceably or with hostile intentions was a matter of doubt - though they were led to expect that he might come with an army to fight them. " (Lyth, 1848-50 : folio 983)

Ainsi, la démarche du Tui Nayau entraîne une cascade de conversions parmi ses sujets et alliés. Si les sujets suivent comme il se doit leur chef, le danger d'instabilité peut venir de l'extérieur, en l'occurrence de Mara Kapaiwai ${ }^{45}$, natif de Bau mais vasu levu de Lakeba. Mara arrive bel et bien à la tête d'une flotte imposante, mais c'est probablement en tant que collecteur de tributs comme il incombe souvent aux vasu, et conformément à la stratégie de Bau en la matière. La paix est préservée ${ }^{46}$. Lyth conclut néanmoins son courrier comme suit :

"It is determined upon by the Tonguese to build a fence for the protection of their wives and families to be ready against any future invasion of Ratu Mara not in anger but in self defence not against Bau or any one but for self preservation. A soro is about to be sent at Bau by the king [Tui Nayau]. I hope you will do your best to lay the whole case on as much as you think proper before Thakombau $[\text { Cakobau }]^{47}$. The soro has been talked of some time \& is therefore not a new idea at all. Lakemba is loyal to Tuinayau \& Tuinayau \& Lakemba are loyal to Bau. God save the king of Lakemba \& the king of Feejee $e^{48}$ too [...] » (Lyth, 1848-50 : folio 988)

Ce courrier montre la dimension politique de la conversion du Tui Nayau. Devenir chrétien alors qu'un allié, et qui plus est un suzerain, n'a pas encore franchi le pas peut être considéré comme un acte de dissidence. Au contraire, suivre son Turaga sur le chemin du lotu est perçu comme un acte de loyauté, voire d'allégeance. La lettre de Lyth explique aussi dans quelle mesure la marge de manœuvre et les moyens d'actions des missionnaires s'inscrivent dans les pratiques traditionnelles. Ainsi, les écrits missionnaires font souvent comme ici référence au soro (demande de pardon rituelle, impliquant une compensation matérielle) pratiqué par les ministres chrétiens et leurs acolytes (voir par exemple Young, $1982: 43$ ).

Ce dernier point ajoute une dimension matérielle importante aux aspects idéologiques et politiques qui semblent présider à la conversion du Tui Nayau. En particulier, ce qui précède a montré en quoi les alliances politiques entre les grands chefs de la région conditionnent l'acceptation et l'incorporation d'idéologies étrangères comme le christianisme à Fidji, avant 1855. Dans le cas des Lau, il semble que le facteur tongien - donc local - soit décisif. Ce sont les missionnaires bénéficiant du meilleur soutien tongien qui réussissent, et l'influence de Tonga se renforce à Lakeba alors que Taliai Tupou, d'ascendance tongienne, se retrouve seul face à des alliances fidjiennes problématiques et privé de son soutien autochtone en la personne de Soroaqali, vasu taukei de Lakeba. Pour ma démonstration, cela confirme le poids de dynamiques déjà à l'œuvre avant l'arrivée des Occidentaux. L'équilibre entre les chefferies de la région est changeant. Ici encore, le facteur occidental semble n'être qu'une dimension de ces transformations.

\section{Somosomo : les échanges nécessaires}

Nous l'avons entraperçu plus haut, les relations entre missionnaires et Fidjiens ne peuvent

44. Wetasau est la forme de Lau (Derrick, $1957: 81$ n12). Les deux orthographes existent dans la littérature.

45. Ce Mara-là - il y a deux Mara parmi les dissidents historiques de Bau (Routledge, 1985 : 50-51) - est le fils de Vuibureta et de Veisaca de Lakeba. Son père est lui-même fils de Banuve et de Ufia, également originaire de Lakeba. Il est exécuté en 1859 sur ordre de Cakobau (Young, 1982 : 46-47 ; Routledge, 1985 : 80).

46. À terme, les tensions religieuses ne se concentrèrent pas sur Bau même. Elles se firent surtout sentir dans les îles de la région de Moala, connectées aux Lasakau de Bau, et à Vanua Balavu, sous influence de Somosomo (Routledge, 1985 : 73). Avec l'aide des Tongiens, y compris celle de Ma’afu (voir note 37), la iValu ni lotu ou guerre de religion qui y fut menée tourna à l'avantage des wesleyens en 1853.

47. En 1849, Cakobau n'est pas encore officiellement Vunivalu, mais son influence à Bau et au-delà est grandissante depuis la fin des années 1830 (Routledge, $1985: 61$ ).

48. Le titre Tui Viti (chef de toutes les Fidji) aurait commencé à être utilisé au cours des années 1830 " par Tanoa " (Routledge, 1985 : 61). Il désigne sans doute déjà Cakobau, dont l'influence est croissante bien avant la fin du règne de son père. 
être réduites aux seuls domaines spirituel ou politique, y compris dans les Lau (Leclerc-Caffarel, $2013: 255-259)^{49}$. De la même façon que marchands et beachcombers ne participaient pas seulement à des transactions commerciales, la dimension matérielle de l'installation missionnaire dans les chefferies fidjiennes est importante. Les enjeux de cette incorporation multiple (de croyances, de schémas de pensée, mais aussi de biens et d'hommes) sont particulièrement sensibles à Somosomo dès la fin des années 1830.

Lorsque les missionnaires wesleyens s'installent à Lakeba (1835), Yavala est le Tui Cakau. Mais la littérature montre que son fils, Tuii ila'ila, est déjà influent dans la chefferie. Il le devient peut-être plus encore à la mort de son frère, Rabici, en juillet 1839 - concomitamment à l'arrivée des révérends Hunt et Lyth à Taveuni (Schütz, 1977 : 146). En janvier 1837, Cargill mentionne une visite du Tui Cakau et de ses fils à Lakeba :

"They were anxious to have Missionnaries and request Bror Cross to accompany them. " (Schütz, 1977 : 92)

Les raisons de cette requête et les arguments en sa faveur sont présents dans la correspondance de Cargill. Le missionnaire prête aux Turaga les propos suivants :

"The Chief of Lakemba is not powerful, - his people are very few and poor, \& he cannot practise what you teach without the consent of his superiors. If you come to us, we will allow our children to be tanght to read on your first arrival, \& we will listen to your doctrine, that we may know if it is true or false, beneficial or useless. " (Schütz, 1977 : 95)

Dans le même temps pourtant, Tuii ila'ila aurait dit :

"Tui Nayau has become a very powerful chief - I am overwhelmed.» (Cargill, 1841 : 150, cité par Reid, $1990: 29)$

Et Yavala de préciser :

"True - everything is true that comes from the white man's country: muskets \& gunpowder are true, \& your religion must be true." (Schütz, 1977 : 95)

Ces propos illustrent une certaine convoitise, engendrée par la présence missionnaire et l'accès aux biens d'importation qu'elle permet (Reid, 1990 : 29). Un parallèle est possible avec ce qui a déjà été noté en marge du commerce du bois de santal. Là encore, il s'agit d'une lutte de pouvoir et d'influence, assujettie à la géopolitique locale. Le Tui Cakau est plus indépendant de Bau que son allié de l'est. Il craint sans doute que son pouvoir ne décroisse au profit de celui du Tui Nayau. Dans le même temps, il aspire à un plus grand accès aux choses venues d'Occident, jusque-là surtout obtenues par le biais d'échanges locaux, y compris via Tonga (Henderson, 1931 : 237).

Cet aspect demeure vivace jusqu'à la fin des années 1840 à Somosomo. On trouve dans les journaux missionnaires de nombreuses références à l'attitude de Tui' ila'ila quant aux biens d'origine occidentale. La plupart dénoncent les demandes répétées du Turaga, jugées excessives - voir par exemple la lettre du révérend Williams datée de mai 1844, évoquant l'intervention du commandant de l'U.S. Exploring Expedition pour les faire cesser (Henderson 1931 : 184-186 n. 65).

Dans le même temps, on constate une insatisfaction grandissante de Tui'ila'ila, et d'autres chefs, face à l'attitude réservée des missionnaires en matière de "dons " (gifts) (par exemple Henderson $1931: 250$ ). Ainsi, en 1839 , Lyth prête à Tui'ila'ila les mots suivants :

"This is a land of chiefs, \& it is our custom to give \& not to sell. Give us your riches, \& we will freely give you food. " (Sahlins, $1993: 858)^{50}$

Ce que le Turaga explique ici est typique de la gestion fidjienne des flux d'échanges (Leclerc-Caffarel, 2013), que l'on pourrait qualifier d'après Sahlins (1972 : 194-195) de réciprocité équilibrée (balanced reciprocity). C'est-à-dire qu'elle implique un rendu pour chaque chose donnée, matérielle ou immatérielle, sans obligation d'immédiateté. En se plaçant sous la protection de chefs locaux les missionnaires s'insèrent dans ce système, combiné à la mise en commun des ressources évoquée en début d'article. Malheureusement, cette réciprocité normale et nécessaire dans le contexte fidjien traditionnel semble mal négociée de part et d'autre de la relation avec les missionnaires, obligeant d'un côté les chefs à « mendier " - en réalité kerekere, à demander ce à quoi ils estiment avoir droit (Sahlins, 1993) - et de l'autre les émissaires chrétiens à " payer ".

Cette inadéquation est contre-productive du point de vue du lotu puisqu'elle attise la résistance de certains Turaga - et donc de leur peuple. Elle entraîne sans doute en partie l'abandon de la mission de Somosomo par les wesleyens en 1847 (Calvert, 2003 : 45). Elle incite en tous cas les missionnaires à tenter un changement de stratégie vers la fin des années 1840. Calvert résume le problème :

"On arriving in the islands, the Missionaries had found it necessary to conciliate the Chiefs and people, and obtain a safe dwelling among them, by the liberal distribution of presents. The practice, which thus began with neces-

49. Outre la dimension matérielle, il faut souligner aussi la pratique cruciale de la médecine par certains missionnaires, dont le révérend Lyth (voir Leclerc-Caffarel, $2013: 248$ ).

50. La référence que Sahlins donne pour cette citation est une entrée du journal de Lyth (1835-42) datée du 30 décembre 1839. Je ne retrouve pas cette phrase à la date indiquée. 
sity, had been continued in compliance with the shameless opportunity of the Chiefs, until it had grown into a burdensome tax, and placed Missionaries upon a false footing, by endangering their spiritual influence over the people, in substituting that which any one might gain by gifts. Among the people themselves, the presentation of a gift without an equivalent in return was an acknowledgment of inferiority and subjection, whence arose another important reason why a clear understanding should be established in this matter. " (Calvert, 2003 : 283)

Calvert se rend à Viwa, déterminé à mettre un terme au système des "dons excessifs" (promiscuous giving), au bénéfice d'une attitude plus conforme aux idées occidentales de l'échange. Son interlocuteur en cette occasion est Cakobau, le futur Vunivalu de Bau :

"When the Missionary's goods arrived from Lakemba, Thakombau went on board the Wesley, accompanied by Mr. Calvert, who, according to the established custom, as a newcomer, presented the Chief with an offering of property from the district he had left, consisting of two large wooden bowls, a bale of sinnet, and two China pigs. These were received very graciously: but on their way to the shore, Thakombau was compelled to listen to one of the lectures he dreaded so much. It was the first step towards the intended reformation and was after this fashion: "[...] I left England originally with one object, and for that alone I have come to live with you in this part of Fiji. My one great object is to have you saved from your sins and their dreadful consequences in the next world [...] While this is the only object I have in view, I am aware that you are destitute of many articles which we have in England, and which would increase your comfort. Some of this I can obtain for you by writing to my friends in England. I shall be glad to do so, as I should like to see you improved and raised in temporal matters. Only, when I send for goods, I have to pay for them, and you must pay for whatever I obtain for you. We give our time and energies to your salvation; but we have not come to supply you with worldly riches. Yet, if you will pay for what you require, we will try to obtain useful articles for you." " (Calvert, 2003 : 283-284)

Les circonstances de ce discours paradoxal montrent que les missionnaires ont une certaine compréhension des us et des coutumes fidjiens, et qu'ils ont les moyens de s'y conformer - quoique parfois difficilement en raison de problèmes d'approvisionnement. Ils s'y refusent sciemment. De fait, ce que Calvert offre à Cakobau n'est autre qu'une relation commerciale - un échange de biens à la manière occidentale - alors même qu'il insiste sur la vocation spirituelle des missionnaires et, implicitement, sur la différence entre eux et d'autres Occidentaux (par exemple marchands, beachcombers). Sur ces points, Calvert et ses collègues se fourvoient au moins de deux façons; d'une part en pensant qu'il est plus approprié de vendre et d'acheter plutôt que de donner et de recevoir dans le contexte fidjien du milieu du XIx ${ }^{e}$ siècle, d'autre part en présumant que les Turaga distinguent nettement le lotu de la valeur maté- rielle et symbolique des biens d'origine occidentale (voir les propos attribués au Tui Cakau Yavala, plus haut).

Les exemples qui précèdent confirment ce que nous avons vu jusqu'ici. Ils montrent que les échanges, en particulier les échanges de biens, sont prégnants dans les interactions entre les Turaga et les Occidentaux pendant la première moitié du XIX ${ }^{e}$ siècle. Pour les premiers, ils sont un moyen essentiel d'intégrer les seconds à leur univers, même si une fois encore on constate que les Occidentaux restent à la marge de la société fidjienne. Ce caractère marginal, voire inadéquat, est plus évident encore lorsque les conceptions économiques ou morales des uns et des autres entrent en conflit - voir aussi plus haut les réticences liées à la strangulation des veuves ou au cannibalisme. Pourtant, l'ensemble de la période 1800-1855 montre que les transactions se succèdent et se renouvellent sans cesse, à la faveur de la capacité d'adaptation de tous les protagonistes. Cette perpétuation est fonction des objectifs de chacun, Fidjiens comme Occidentaux. Ce qui suit montre que leurs transactions revêtent parfois un caractère de nécessité. C'est le cas de la conversion au lotu de Cakobau, jugée décisive pour l'avenir religieux de l'archipel, et cruciale en termes d'alliances pour le Vunivalu.

\section{La conversion de Cakobau : le contexte de la guerre entre Bau et Rewa}

L'hostilité de Bau quant aux stratégies missionnaires n'est pas seulement le fait des Turaga appartenant au clan des Vunivalu (Tui Kaba), quoique sans doute en partie inféodée à leur choix. Ainsi, Lyth cite un chef de Bau, sans précision, dans ses Réminiscences fidjiennes et tongiennes:

"You come here \& you will only buy and sell, and we hate buying. [When] we ask you for a thing you say no. If a Feejeean said no, we sh ${ }^{d}$ kill him, don't you know that. We are a land of Chiefs. We have plenty of riches. When we come to Somosomo, we have plenty of pigs, and in Lakemba \& in every koro [village]. We have them without buying. We hate buying and we hate the lotu. We will never lotu. » (Lyth, s.d. : 74-75)

Au tournant des années 1840, Cakobau semble lui percevoir l'obtention de biens importés comme la seule raison valable de se convertir. Mais, il subordonne ce désir au respect de la hiérarchie fidjienne. Ainsi, suite à la conversion de son conseiller Verani, le neveu du Tui Viwa déjà mentionné, M. Wallis lui prête ces propos :

"Verani send us the riches that you have obtained by 'lotuing.' They belong to us [Cakobau/Vunivalu]. Why have you 'lotued?' What have we done, that you have become angry with us, and left us?" (Wallis, 1851 : 71) 
La conversion de Verani est un affront pour Cakobau, d'autant plus grand que Wallis écrit avoir entendu le futur Vunivalu dire :

"I told him to wait a little, and then we would 'lotu' together. " (Wallis, 1851: 70)

Mais si en 1845 Cakobau envisage sa propre conversion, c'est sans doute en termes de monnaie d'échange et au service de sa propre emprise sur les partenariats en jeu. Ainsi :

"He tells Mr. W. that if he 'lotues,' he will burn his 'beech de mer' houses, and forbid the natives to fish." (Wallis, $1851: 82$ )

Quelques années plus tard la réaction de Cakobau au discours de Calvert déjà cité - telle que ce dernier la transcrit - peut donc paraître surprenante :

"Thakombau listened complacently [...] and said he was glad to know the right plan, and should like to be informed of what was expected in payment for any articles he might hereafter desire. A decisive and important step was thus taken, which made it easier to resist the perpetual begging of smaller people. Yet, in many cases, it was still hard to refuse [...] Nevertheless, though it was still necessary to make occasional presents, the more reserved plan was found to answer; for the people learned to value what they worked for, and gained self-respect in being rid of a system which pauperized them. " (Calvert, 2003 : 284-285)

Dans cet extrait, Calvert néglige la réciprocité intrinsèque à la chefferie ; et il omet de considérer son interlocuteur comme un véritable stratège politique.

En 1845, l'avantage est du côté de Cakobau. La guerre entre Bau et Rewa commence à peine. L'ascendant de Cakobau sur les Fidji est solide. En décembre, il met notamment à mort le Roko Tui Dreketi Banuve - homonyme du Vunivalu de Bau évoqué en deuxième partie, et porteur du titre principal de Rewa. Le titre échoit à Cakonauto (Routledge, 1985: 68, 82), mieux connu des Européens sous le nom de Phillips (Eagleston, 1834). Il est vasu des Tui Kaba (le mataqali des Vunivalu de Bau). En bons termes avec Cakobau comme avec les Occidentaux, son règne sur Rewa semble à l'avantage des Vunivalu de Bau (Routledge, 1985: 69). Le Tui Cakau Yavala vient aussi de mourir, laissant la place à son inoffensif cousin (Ralulu) à Somosomo, en paix avec Bau depuis 1842. Tui'ila'ila reste le leader officieux de la chefferie, mais il semble qu'il ait aussi eu des relations harmonieuses avec Cakobau pendant les dernières années de sa vie (idem: 79). Enfin, sa propre conversion fait l'objet de toutes les attentions missionnaires, alors même que la main d'œuvre qu'il peut four- nir aux marchands de bêches-de-mer à Ovalau et Viwa reste une monnaie d'échange précieuse. En 1854 néanmoins, les forces en présence ont changé au détriment du Vunivalu. Les Occidentaux basés à Levuka ont gagné en autonomie, et après une dispute avec Cakobau se sont alliés au Roko Tui Dreketi. Cakonauto/Phillips est mort en 1852. Qaraniqio lui a succédé. D'après Routledge (1985), il est vasu des Vusaratu (le mataqali du Roko Tui Bau), quoique peut-être apparenté par sa mère plutôt à la noblesse locale de Bau (mataqali Nabaubau) pour Sahlins (2004). Il est dans les deux cas vasu d'un clan rival des Tui Kaba (mataqali des Vunivalu de Bau), en plus d'être un ennemi d'enfance de son frère classificatoire, Cakobau, également vasu de Bau puisque leurs mères seraient "sœurs". Les Turaga de Rewa et les gens d'Ovalau ont organisé le siège de Bau, privant la chefferie de ses contacts avec les Occidentaux, de son approvisionnement en armes et en munitions, et de précieux soutiens. Mara Kapaiwai, déjà cité, a aussi rejoint la cause de Qaraniqio. Son rang lui permet de prétendre au trône de Bau (Routledge 1985 : 80-82). Pendant ce temps, à l'est, Ma'afu est devenu le gouverneur des Tongiens de Fidji. Il a gagné en influence dans la région de Moala, jusque-là sous le joug de Bau, et s'apprête à faire de même à Vanua Balavu avec l'accord de Tui' ila'ila (Reid, $1990: 41-42)^{51}$.

Rewa bénéficie en outre du soutien de J.B. Williams, le premier consul des États-Unis (1846$60)$. Ce personnage est un des premiers Occidentaux "indépendamment actifs " à Fidji, aux côtés de D. Whippy (Routledge, 1985 : 69-71). À la différence de ce dernier cependant, et en dépit d'une brève alliance entre les deux hommes, Williams apparaît dans la littérature comme un personnage sans scrupules, avide et peu préoccupé du sort des Fidjiens (Campbell, 1998 : 6667). À Fidji, il s’adonne à la spéculation foncière et prend part à la guerre entre Rewa et Bau en faisant peser sur Cakobau des réclamations, des dettes et des menaces de plus en plus importantes, suite notamment au saccage de ses propriétés par des insulaires et en vertu du titre Tui Viti alors attribué à Cakobau de façon virtuelle, puisque en dépit d'une influence accrue des Vunivalu de Bau depuis le début du siècle (voir plus haut) il n'est pas encore question d'unité à Fidji au début des années 1850 , et certainement pas sous le patronage de Cakobau. Quelques années plus tôt, Williams avait déjà vendu à Cakobau un bateau de facture européenne pour plus de six fois sa valeur en huile de coco (Kenny, 1956 : 9-10). Plusieurs autres ressortissants américains, dont Whippy, viennent ajouter aux réclamations de Williams. En 1855, ce qu'on appelle la "dette américaine " atteint \$43,686 (Campbell, 1998 : 67). 
En avril 1854, un mois après un grave incendie à Bau (Waterhouse, 1997 : 159-160), suivi d'une défaite à Kaba (idem: 165), Cakobau est fortement endetté et en mauvaise posture vis-à-vis du Roko Tui Dreketi et de ses autres opposants. Il reçoit alors une lettre du roi de Tonga (Taufa'ahau/ George Tupou) lui enjoignant de se convertir. Si l'on en croit Waterhouse, le Tui Tonga, jusquelà évasif quant à l'aide qu'il pouvait apporter au Vunivalu la conditionnerait maintenant de façon explicite : "I wish, Thakombau, you would lotu " (ibid.: 169). Affecté par les événements du début de l'année 1854 - dont la mort de Tui'ila'ila -, considérablement affaibli par le siège de Rewa, apparemment délaissé par ses propres dieux, et pressé par ses alliés potentiels de prendre position quant au lotu (Waterhouse, 1997 : 176177), Cakobau semble n'avoir guère le choix. Il se convertit le 30 avril 1854 (idem : 178-179).

Comme ses pairs, Cakobau s'est donc adapté aux circonstances. Il canalise tant bien que mal les forces extérieures au profit de son emprise politique sur les événements. 1855 est pour lui une année victorieuse. Elle commence par la mort (naturelle) de Qaraniqio, rapidement suivie de la célèbre bataille de Kaba (7 avril) qui entérine la victoire du Vunivalu et des Tongiens sur Rewa et les rebelles de Bau (Routledge, 1985 : 86). Le pouvoir de Bau restauré, la conversion du Vunivalu en entraîne bien d'autres. Mais alors que l'influence des Occidentaux dans les affaires fidjiennes est de plus en plus tangible, la tactique de Cakobau reste conforme à celle de ses prédécesseurs, plus proches des négociants qui passent ou s'installent dans les îles que des missionnaires qui veulent tant les transformer (Routledge, $1985: 88$ ).

De la même façon, Cakobau et la plupart des Turaga cités plus haut décident de s'en remettre aux Anglais plutôt qu’aux Américains dès les années 1860, et jusqu'à la Cession de la souveraineté fidjienne à la reine d'Angleterre en 1874. En plus des dissensions morales qui existent entre les chefs fidjiens et les voyageurs occidentaux de tous types, les officiels américains, dont Ch. Wilkes et surtout J.B. Williams, accablent les Turaga de responsabilités pénales qui n'avaient pas cours jusque-là (Wilkes, 1845 ; Routledge, 1985 : 71). Les Anglais apparaissent comme un rempart aux difficultés que cela engendre, sur fond de rivalité larvée entre les États-Unis et la Grande-Bretagne (McIntyre, 1960). En règle générale, les officiels anglais comme le capitaine Denham, puis les gouverneurs britanniques, se montrent plus attentifs aux usages locaux et entrent plus volontiers que leurs homologues américains dans des relations d'échanges avec les Turaga, comparables aux pratiques traditionnelles (Routledge, 1985 : 84 ; Herle \& Carreau, 2013).

En 1860, une première proposition de Cession est examinée par le gouvernement britannique qui la refuse. Quelques années plus tard néan- moins la dette américaine est soldée (McIntyre, 1960 ; Routledge, 1985: 117-120) et la Cession à la couronne d'Angleterre est entérinée. Après 1874, une partie des conflits entre Turaga et créanciers américains se reversent dans une bataille juridique entre les États-Unis et l'Angleterre. Elle ne prend fin que dans les années 1920. La question de la propriété des terres fidjiennes est au cour des débats (Fromageot, 1924). Elle est aussi prépondérante dans certaines problématiques coloniales et postcoloniales à Fidji (par exemple France, 1969; Kaplan, 1988; Kelly et Kaplan, 2001 : 161), témoignant de la différence intrinsèque qui subsiste jusqu'à nos jours, du point de vue fidjien, entre les autochtones (itaukei) et les étrangers (vulagi), y compris aujourd'hui les Fidjiens d'origine indienne qui descendent de travailleurs employés par le gouvernement colonial dans les plantations à la fin du XIX ${ }^{e}$ siècle.

\section{Conclusion}

L'objectif de cet article était d'appréhender les relations entre les Fidjiens et les Occidentaux du point de vue des chefs fidjiens, de 1800 à 1855 . Il s'est appuyé sur l'exemple des chefferies de Bua, Bau, Lau et Somosomo, avec des références subsidiaires à Rewa et Viwa. Dans le même temps, il a fait appel aux récits et aux collections compilés par plusieurs types de voyageurs occidentaux, marchands et missionnaires surtout, mais aussi officiers de marine, consuls et premiers colons (settlers) installés dans les îles. Ces cas d'étude, choisis pour leur pertinence historique et pour leurs points commun structurels, ont permis d'illustrer la pluralité des relations entre les Fidjiens et les Occidentaux, prises dans un ensemble de dynamiques endogènes et exogènes.

En dépit de cette complexité, de grands traits peuvent être dégagés. Fondamentalement, les principes de réciprocité et de complémentarité inhérents à l'exercice du pouvoir à Fidji sont aussi à l'œuvre dans la gestion des incursions étrangères par les Turaga. Ce sont des partenariats que les chefs semblent chercher auprès des voyageurs occidentaux, et des modèles préexistants sont utilisés pour canaliser et intégrer certains EuroAméricains au monde fidjien. Cependant, ces visiteurs restent toujours en marge du système ; ils demeurent des étrangers (kai tani) qui vont et viennent. Pour les marchands et les officiers de marine, Fidji n'est souvent qu'une escale au cours de leurs voyages. Quelques partenariats au long cours se créent néanmoins, entre Naulivou et Savage ou bien Tanoa et Whippy par exemple. Par définition, les missionnaires tendent vers des relations plus pérennes, mais c'est à condition de profondes transformations chez leurs hôtes. 
En dépit de ces divergences de perception, les Occidentaux deviennent des partenaires d'échange importants pour les Turaga pendant la première moitié du XIX ${ }^{\mathrm{e}}$ siècle - quoique différemment selon la situation géographique et politique de chaque chefferie et les ambitions de chaque chef. Les objets venus d'au-delà des mers, jusque-là surtout obtenus via des échanges locaux, notamment auprès des Tongiens, prennent une importance nouvelle. Ils affluent en larges quantités dans les îles dès les années 1800 , comme le montrent les récits des EuroAméricains impliqués dans le commerce du bois de santal, puis des bêches-de-mer. En particulier, l'ivoire, les armes à feu, et dans une moindre mesure les objets en métal et les pièces de tissu, deviennent des objets d'échange et de valeur locaux (iyau). Leur accumulation marque le rang. Et en raison de leur valeur symbolique, certains matériaux comme l'ivoire de cachalot et le tissu rouge entrent dans la composition de ce qu'Hooper (2013) appelle des regalia. Il s'agit par exemple de pectoraux composites, notamment observés à Bau et à Somosomo (Wilkes, 1845, III : 58-59; Henderson, $1931: 187)$, et de colliers en dents de cachalot et tissu rouge (Dodge, 1972 : 187). Ces objets, remarqués par les visiteurs occidentaux, sont globalement absents des collections muséales avant 1855 ; de même pour les fusils incrustés d'ivoire. Cette inaliénabilité témoigne de leur valeur aux yeux des chefs fidjiens (voir Leclerc-Caffarel, 2013).

Selon la tradition fidjienne, c'est à l'aune d'échanges que l'on mesure la puissance et la bonne volonté d'un allié, réel ou potentiel, et que l'on crée avec lui un partenariat plus ou moins solide. Parmi les nombreux indices qui attestent du mana d'un Turaga, c'est-à-dire de son efficacité dans l'exercice de sa fonction, on trouve ses insignes de rang (dont les regalia susmentionnés) mais aussi ses succès militaires et son aptitude à fournir à son peuple, à ses alliés ou, le cas échéant, à son suzerain des choses précieuses (iyau) et de la nourriture. Parmi ces offrandes, la chair humaine, nourriture des dieux et des Turaga, est à part, tout comme les femmes qui circulent dans le sens inverse des bokola (victimes sacrificielles). Le parallèle que Sahlins souligne, au début du XIX $^{e}$ siècle, entre la quantité de dents de cachalot en circulation et l'augmentation de la consommation cannibale à Fidji (1983: 90-91) illustre la reconfiguration de l'équilibre politique qui a lieu dans l'archipel. Cette dernière se traduit par la montée en puissance de certains Turaga en contact avec les Occidentaux, comme les Vuniva$l u$ de Bau. Notons que les armes à feu, désormais fréquentes sur les champs de bataille fidjiens, participent aussi à l'accroissement du nombre de victimes sacrificielles. De façon générale, c'est la capacité d'accumulation (de bokola, de victoires, d'iyau, d'alliés, etc.) de certains grands chefs, et donc leur puissance, qui est accrue. Si le facteur occidental, pluriel, n'est qu'un des aspects de cette restructuration du pouvoir, en termes militaires et en matière d'échanges le rôle des objets véhiculés par les Occidentaux paraît, lui, capital. À l'évidence, il ne faut pas occulter les motivations des Euro-Américains, et leurs propres réussites dans la manipulation de la géopolitique fidjienne du $\mathrm{XIX}^{\mathrm{e}}$ siècle. Mais, il convient de garder en tête la dimension fondamentalement matérielle et diplomatique de ces relations pour les chefs fidjiens avant 1855. Dans tous les cas précités, il est d'abord question d'échanges, de partenariats et de rivalités locales pour les Turaga. Et cela explique notamment leur convoitise pour les biens importés, le dépit qu'engendrent les échecs et les déconvenues dans les relations avec les Euro-Américains et l'attitude apparemment versatile de certains souverains vis-à-vis des Occidentaux.

\section{BIBLIOGRAHIE}

Broc Numa, 2003. Dictionnaire illustré des explorateurs et des grands voyageurs français $d u$ XIX siècle, Paris, éd. du cTHs.

Brewster A., 1937. King of the Cannibal Isles: A tale of early life and adventure in the Fiji Islands, Londres, Robert Hale \& Company.

Calvert James, 2003 [1858]. Fiji and the Fijians vol. II: Mission History, Suva, Fiji Museum.

Campbell Ian, 1980. The Historiography of Charles Savage, Journal of the Polynesian Society, 89 (2), pp. 143-166.

-, 1998. Gone Native in Polynesia: Captivity Narratives and Experiences from the South Pacific, Westport, Connecticut, Greenwood Press.

Capell Arthur, 1941. A New Fijian Dictionary, Sydney, Australian Medical Publishing Company Limited.

CARY William, 1998 [1887]. Wrecked on the Feejees, Fairfield, Washington, Ye Galleon Press.

Clunie Fergus, 1983a. The Fijian Flintlock, Domodomo 1 (3), pp. 102-122.

-, 1983b. Tanoa Visawaqua's Breastplate, Domodomo 1 (3), pp. 123-125.

-, 2003a [1986]. Yalo i Viti: A Fiji Museum Catalogue, Suva, Fiji Museum.

-, 2003b [1977]. Fijian Weapons and Warfare, Suva, Fiji Museum.

—, 2013. Tapua: "Polished Ivory Shrines" of Tongan Gods, Journal of the Polynesian Society 122 (2), pp. 161-203. 
DAмm Hans, 1975. Scheibenförmiger Brustschmuck aus Pottwalzahnen, Fidschi-Inseln, Abhandlungen und Berichte des Staatlichen Museums für Völkerkunde Dresden 34, pp. 23-54.

Deane Wallace, 1921. Fijian Society: or the Sociology and Psychology of the Fijians, Londres, Macmillan.

Derrick Ronald, 1957. A History of Fiji (3e éd.), Suva, Government Press.

Dillon Peter, 1829. Narrative of a Successful Voyage to the South Seas, London, Hurst, Chance.

Dodge Ernest, 1972. A William Lockerby Manuscript in the Peabody Essex Museum, Journal of Pacific History 7, pp. 182-188.

Dumont D’Urville Jules, 1841-54. Voyage au pôle sud et dans l'Océanie sur les corvettes l'Astrolabe et la Zélée, exécuté pendant les années 1837-1838-1839-1840, sous le commandement de J. Dumont d'Urville, capitaine de vaisseau, publié par ordonnance de Sa Majesté, Paris, Gide.

Faure Félix, 1929. John Hunt: missionnaire aux îles Fidji (1812-1848), Paris, Société des missions évangéliques.

France Peter, 1969. The Charter of Land: Custom and Colonization in Fiji, Melbourne, Oxford University Press.

GaTTy Ronald, 2009. Fijian-English Dictionary: with notes on Fijian culture and natural history, Suva, R. Gatty.

Geraghty Paul, 1983. The History of the Fijian Languages, Honolulu, University of Hawaii Press.

Henderson George (ed.), 1931. The Journal of Thomas Williams, Sydney, Angus \& Robertson.

-, 1933. The Discoverers of the Fiji Islands: Tasman Cook, Bligh, Wilson, Bellingshausen, London, J. Murray.

Herle Anita et Lucie Carreau (eds), 2013. Chiefs and Governors: Art and Power in Fiji, Cambridge, Museum of Archaeology and Anthropology, University of Cambridge.

Hocart Arthur, 1929. Lau Islands, Fiji, Honolulu, Bernice P. Bishop Museum.

—, 1970 [1936]. Kings and Councillors: An essay in the Comparative Anatomy of Human Society, R. Needham (eds), Chicago, University of Chicago Press.

Hoffman Cleaver Anne et Jeffrey Stann (eds), 1998. Voyage to the Southern Ocean: the Letters of Lieutenant William Reynolds from the U.S.
Exploring Expedition, 1838-1842, Annapolis, Naval Institute Press.

Hooper Steven, 1982. A study of valuables in the chiefdom of Lau, Fiji, Thèse doctorale, University of Cambridge, St. John's College, Faculty of Archaeology and Anthropolgy.

—, 1996. Who are the Chiefs? Chiefship in Lau Eastern Fiji, in R. Feinberg, R. Firth \& K. Watson-Gegeo (eds), Leadership and Change in the Western Pacific, Londres, Athlone Press, pp. 239-271.

-, 2013. "Supreme among our Valuables": Whale Teeth Tabua, Chieftainship and Power in Eastern Fiji, Journal of the Polynesian Society 122 (2), pp. 103-160.

Im Thurn Everard et Leonard Wharton (eds), 1925. The journal of William Lockerby: sandalwood trader in the Fijian Islands during the years 1808-1809, with an introduction \& other papers connected with the earliest European visitors to the Islands, Londres, Hakluyt Society.

Kaeppler Adrienne, 1978. Exchange Patterns in Goods and Spouses: Fiji, Tonga and Samoa, Mankind 11 (3), pp. 246-252.

Kaplan Martha, 1988. The Coups in Fiji: Colonial Contradictions and the Post-Colonial Crisis, Critique of Anthropology 8 (3), pp. 93-116.

Kelly, John et Martha Kaplan, 2001. Represented Communities: Fiji and World Decolonization, Chicago, University of Chicago Press.

Kenny Robert (ed.), 1956. The New Zealand Journal, 1842-1844 of John B. Williams of Salem, Massachusetts, Salem, Peabody Essex Museum.

Leclerc-Caffarel Stéphanie, 2013. Exchange relations between Fijians and Euro-Americans (1774-1854), with reference to museum collections, thèse doctorale, University of East Anglia (Norwich), School of World Art Studies and Museology.

Maude Harry, 1964. Beachcombers and Castaways, Journal of the Polynesian Society 73 (3), pp. 254-293.

McIntyre W.D., 1960. Anglo-American Rivalry in the Pacific: The Bristish Annexation of the Fiji Islands in 1874, Pacific Historical Review 29 (4), pp. 361-380.

Pauwels Simonne, 2015. The Vasu Position and the Sister's Mana: The Case of Lau (Fiji), in Living Kinship in the Pacific, Oxford, Berghahn, pp. 143-165. 
QuaIn Buell, 1948. Fijian Village, Chicago, University of Chicago Press.

Reid A.C., 1990. Tovata I \& II, Suva, Fiji Museum.

Roth George \& George Milner, 1973. Fijian way of life, Londres, Oxford University Press.

Ravuvu Asesela, 1983. Vaka i Taukei: the Fijian way of life, Suva, University of the South Pacific.

Routledge David, 1985. Matanitu: the Struggle for Power in early Fiji, Suva, University of the South Pacific.

SAHuIns Marshall, 1962. Moala: Culture and Nature on a Fijian Island, Ann Arbor, University of Michigan Press.

—, 1972. Stone Age Economics, Chicago, AldineAtherton.

-, 1983. Raw Women, Cooked Men, and Other "Great Things" of the Fiji Islands, in P. Brown \& D. Tuzin (eds), The Ethnography of Cannibalism, Washington, Society for Psychological Anthropology, pp. 72-93.

—, 1985. Islands of History, Chicago, University of Chicago Press.

-, 1993. Cery Cery Fuckabebe. American Ethnologist 20 (4), pp. 848-867.

—, 2004. Apologies to Thucydides: Understanding History as Culture and Vice Versa, Chicago, University of Chicago Press.

Schütz Albert (ed.), 1977. The diaries and correspondence of David Cargill, 1832-1843, Canberra, Australian National University Press.

Seemann Berthold, 1862. Viti: an Account of a Government Mission to the Vitian or Fijian Islands 1860-1861, Cambridge, MacMillan and Co.

Tatawaqa Pita, 1913. Charlie Savage, Transactions of the Fijian Society, pp. 1-4.

Thomas Nicholas, 1991. Entangled Objects: Exchange, Material Culture, and Colonialism in the Pacific, Londres, Harvard University press.

Thornley Andrew, 1996. The Legacy of Siloam: Tahitian Missionaries in Fiji, in Munro \& Thornley (eds), The Convenant Makers: Islander Missionaries in the Pacific, Suva, University of the South Pacific, pp. 91-114.

-, 2000. The Inheritance of Hope: John Hunt, Apostle of Fiji, Suva, University of the South Pacific.
-, 2002. Exodus of the I Taukei: The Wesleyan Church in Fiji: 1848-74, Suva, University of the South Pacific.

-, 2005. A Shaking of the Land: William Cross and the Origins of Christianity in Fiji, Suva, University of the South Pacific.

Toganivalu Deve, 1917. Ai Yau Kei Na Yaya Vaka Viti: Fijian Property and Gear, Transactions of the Fijian Society, pp. 1-18.

Toren Christina, 1994. All Things Go in Pairs, or the Sharks Will Bite: The Antithetical Nature of Fijian Chiefship, Oceania 64 (3), pp. 197-216.

Wallis Mary, 1851. Life in Feejee, or Five Years Among the Cannibals, Boston, William Heath.

Waterhouse Joseph, 1997 [1866]. The King and People of Fiji, Honolulu, University of Hawaii Press.

WiLkes Charles, 1845. Narrative of the United States exploring expedition during the years 1838, 1839, 1840, 1841, 1842, Philadelphia, Lea $\&$ Blanchard.

Young John, 1982. The Response of Lau to Foreign Contact: An Interdisciplinary Reconstruction, Journal of Pacific History 17 (1), pp. 29-50.

\section{ARCHIVES}

Eaglestone John, 1834. Lettre, Canberra, Pacific Manuscript Bureau, Mf. 225.

Lockerby William, 1813. Directions for the Fegee or Sandalwood Islands, Canberra, Pacific Manuscript Bureau, Mf. 225.

Lyтн Richard, 1848-50. Journal, Sydney, Mitchell Library, Mf. B536-1.

—, 1836-42. Journal, Sydney, Mitchell Library, Mf. B533.

-, S.D. Tongan and Fijian reminiscences, Sydney, Mitchell Library, Mf. B549.

Osborn J. Warren, 1833-35. Journal of the Emerald, Canberra, Pacific Manuscript Bureau, Mf.225.

Rocquemaurel Gaston (de), 1837-40. Journal, Paris, Archives Nationales, Mf. 5JJ 144.

Toganivalu Deve, s.d. An Island Kingdom: The history and customs of the Bauans, and their influence on Fijian Society and Government, Suva, National Archives of Fiji. 


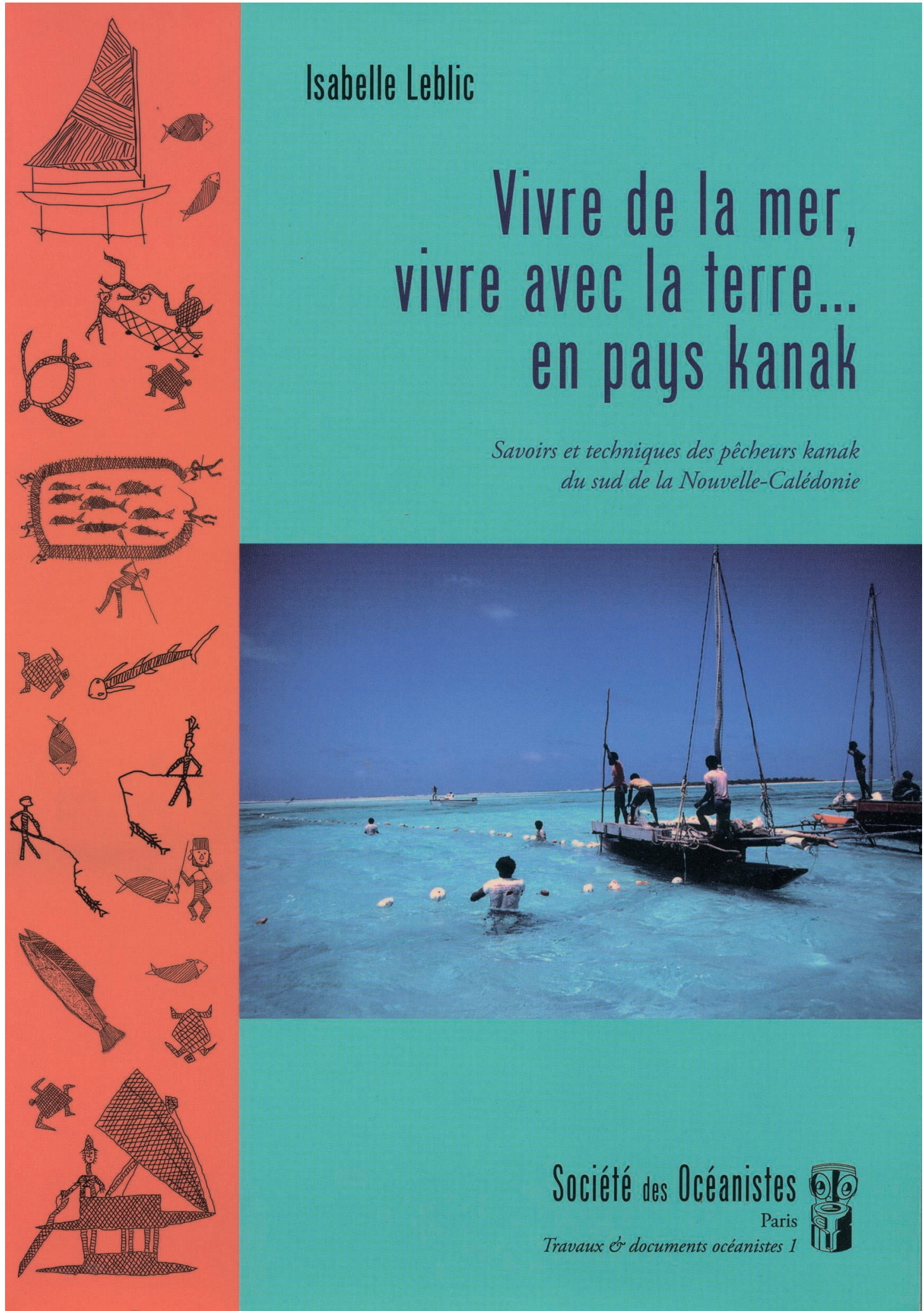

2008, 288 p., bibliogr., glossaires, index, plus de 600 ill. en noir \& blanc ou en couleur - $38 €$. En vente sur http://oceanistes.org/oceanie/spip.php?rubrique29 avec paiement en ligne et en version électronique sur http://books.openedition.org/sdo/594-29,99€. 